\title{
Improving the neutrino mass hierarchy identification with inelasticity measurement in PINGU and ORCA
}

\author{
Mathieu Ribordy ${ }^{1, *}$ and A. Yu. Smirnov ${ }^{2}, \dagger$ \\ ${ }^{1}$ High Energy Physics Laboratory, École Polytechnique Fédérale, CH-1015 Lausanne, Switzerland \\ ${ }^{2}$ The Abdus Salam International Centre for Theoretical Physics, I-34100 Trieste, Italy
}

(Dated: November 5, 2018)

\begin{abstract}
Multi-megaton scale under ice and underwater detectors of atmospheric neutrinos with few GeV's energy threshold (PINGU, ORCA) open up new possibilities in the determination of neutrino properties, and in particular the neutrino mass hierarchy. With a dense array of optical modules it will be possible to determine the inelasticity, $y$, of the charged current $\nu_{\mu}$ events in addition to the neutrino energy $E_{\nu}$ and the muon zenith angle $\theta_{\mu}$. The discovery potential of the detectors will substantially increase with the measurement of $y$. It will enable (i) a partial separation of the neutrino and antineutrino signals; (ii) a better reconstruction of the neutrino direction; (iii) the reduction of the neutrino parameters degeneracy; (iv) a better control of systematic uncertainties; (v) a better identification of the $\nu_{\mu}$ events. It will improve the sensitivity to the CP-violation phase. The three dimensional, $\left(E_{\nu}, \theta_{\mu}, y\right), \nu_{\mu}$-oscillograms with the kinematical as well as the experimental smearing are computed. We present the asymmetry distributions in the $E_{\nu}-\theta_{\mu}$ plane for different intervals of $y$ and study their properties. We show that the inelasticity information reduces the effect of degeneracy of parameters by $30 \%$. With the inelasticity, the total significance of establishing mass hierarchy may increase by $(20-50) \%$, thus effectively increasing the volume of the detector by factor $1.5-2$.

PACS numbers: $14.60 . \mathrm{Pq}$
\end{abstract}

\section{INTRODUCTION}

Multi-megaton scale atmospheric neutrino detectors with few GeV's energy threshold have an enormous and largely unexplored physics potential. These detectors are sensitive to the oscillatory patterns due to the 1-3 mixing in the neutrino energy - zenith angle $\left(E_{\nu}-\theta_{\nu}\right)$ plane. The patterns have several salient features, which include the MSW resonance peaks due to oscillations in the mantle $\left(E_{\nu} \sim 6 \mathrm{GeV}\right)$ and the core $\left(E_{\nu} \sim 4 \mathrm{GeV}\right)$ as well as the parametric enhancement ridges at $E_{\nu} \sim(4-12)$ $\mathrm{GeV}$ which are realized for the core crossing neutrino trajectories (see [1] for detailed description and 2] for recent review and references). The patterns differ for neutrinos and antineutrinos and strongly depend on the type of neutrino mass hierarchy. In particular, the indicated features appear in the neutrino channels in the case of normal mass hierarchy $(\mathrm{NH})$ and in the antineutrino channels in the case of inverted hierarchy (IH) (in the two neutrino approximation, inversion of the mass hierarchy is equivalent to switching the neutrino and antineutrino oscillation patterns). This opens up a possibility to establish the neutrino mass hierarchy and also to measure the deviation of the 2-3 mixing from maximal as well as the 1-3 mass splitting. Once the hierarchy is established, one can consider a possibility to measure the CP-violation phase.

Multi-megaton detectors are expected to record of the

*Electronic address: mathieu.ribordy@epfl.ch

${ }^{\dagger}$ Electronic address: smirnov@ictp.it order of $10^{5}$ events a year. Such a large statistics allows, in principle, to compensate shortcomings related to flavor identification of events and reconstruction of their energy and angular characteristics. With so high statistics, one can select some particular events in certain kinematical regions, which are most sensitive to a given neutrino parameter, thus reducing effect of degeneracy of parameters, etc.

PINGU (Precision IceCube Next-Generation Upgrade) 3], the IceCube DeepCore [4] augmented with a denser instrumentation in its center, and ORCA (Oscillation Research with Cosmics in the Abyss) [5] projects are possible future realizations of these Multi-megaton scale detectors.

A simplified estimation of the sensitivity to the mass hierarchy of the DeepCore (DC) experiment has been performed in 6]. Due to the high energy threshold $(>10$ $\mathrm{GeV}$ ), DC has a low sensitivity to the resonance pattern and therefore to the hierarchy. The sensitivity of DC to deviation of the 2-3 mixing from maximal has been explored in [7].

The idea to send a neutrino beam from Fermilab to PINGU to determine the mass hierarchy has been elaborated in 8 .

A possibility to use PINGU and the atmospheric neutrino flux for the identification of the neutrino mass hierarchy $(\mathrm{MH})$ and search for the $\mathrm{CP}$ violation effects was recently explored in 9. The strategy is based on the measurement of the $E_{\nu}-\theta_{\nu}$ distribution of the sum of muon neutrino and antineutrino events. The smearing of the distribution over $\theta_{\nu}$ and $E_{\nu}$ has been performed that takes into account accuracy of reconstruction of the neutrino energy and direction. 
The estimator of discovery potential, $S$ (the hierarchy asymmetry), has been introduced [9], which allows one to make quick evaluation of sensitivities of the detector to neutrino mass hierarchy as well as to other parameters. For $i j$-bin in the reconstructed neutrino energy $(i)$ and zenith angle $(j)$, the asymmetry is defined as

$$
S_{\nu, i j}=\frac{N_{\nu, i j}^{\mathrm{IH}}-N_{\nu, i j}^{\mathrm{NH}}}{\sqrt{N_{\nu, i j}^{\mathrm{NH}}}} .
$$

Here $N_{\nu, i j}^{\mathrm{NH}}$ and $N_{\nu, i j}^{\mathrm{IH}}$ are the numbers of events in the $i j$-bin for the normal and the inverted mass hierarchies correspondingly. The moduli of the asymmetry, $\left|S_{\nu, i j}\right|$, gives the statistical significance for the identification of the mass hierarchy. The asymmetry allows one to explore in a transparent way the dependence of sensitivities on the experimental energy and angular resolutions, on degeneracies of parameters and on various systematic errors. In [9] it was shown that the hierarchy can be established at $(3-10) \sigma$ level after 5 years of operation of PINGU depending on the energy and angular resolutions and on the size of the systematic error.

A final answer concerning the sensitivity should follow from the detailed Monte Carlo simulation of the distributions of events. That should take into account realistic parameters of the detectors after their geometries are determined. Then, in a simple approach, the sensitivity can be obtained from the fit of the simulated distributions with the distributions computed for the cases of normal and inverted mass hierarchies. The fit can be done using Poisson statistics without binning. Results of a "toy" Monte Carlo study for large volume detectors have been presented in 10]. The physics potential of PINGU and ORCA was further explored in [11] and 10.

As it was discussed in [9], several factors dilute the significance of the $\mathrm{MH}$ identification, although at the probability level the effect of inversion of the hierarchy is of the order 1 . Indeed,

(i) the hierarchy asymmetry has opposite signs in different kinematical regions. Therefore smearing over the angle and energy, $E_{\nu}-\theta_{\nu}$, leads to a substantial decrease of the observable effect. The smearing originates from finite energy and angular resolutions of the detector (experimental smearing) and due to difference of the neutrino and muon directions (kinematical smearing);

(ii) the hierarchy asymmetry has different signs for neutrinos and antineutrinos. Therefore summing up the neutrino and antineutrino signals leads to a partial cancellation of the effect;

(iii) the presence of both $\nu_{\mu}$ and $\nu_{\mathrm{e}}$ flavors in the original atmospheric neutrino flux leads, in general, to a suppression of oscillation effects. The suppression becomes weaker at high energies, where the $\nu_{e}$ flux is small;

(iv) current uncertainties of the oscillation parameters, such as $\Delta m_{32}^{2}$ and $\theta_{23}^{2}$ further reduce identification power, since the effect of inversion of the mass hierarchy can be partly mimicked by changes of these parameters; (v) the sample of $\nu_{\mu}$ events is contaminated by contributions from $\nu_{\tau}$ and $\nu_{e}$ charged current (CC) interactions and neutral current (NC) interactions of all neutrino species. In particular, $\nu_{\tau}$ 's generated via oscillations produce tau leptons, which decay in $18 \%$ cases into muons, thus appearing as $\nu_{\mu}$ events. Also $\nu_{e}$ and $\mathrm{NC}$ interactions can mimic $\nu_{\mu}$ events due to muon - pion misidentification. These events produce an additional effective smearing of the oscillatory pattern.

All this renders the quest of the neutrino mass hierarchy difficult. Some (probably modest) developments of technology are required. This includes the selection of certain geometry of the detector, the upgrade of the optical modules, further developments of the time analysis of events, etc. On the other hand, some particular ways to analyse the information obtained can also improve the sensitivity.

In this connection, we explore improvements of the sensitivity to the neutrino $\mathrm{MH}$ due to the measurement of the inelasticity, $y \approx 1-E_{\mu} / E_{\nu}$, of the charged current $\nu_{\mu}$ events. As we will show, this new ingredient in the analysis enables us to alleviate some of the problems mentioned above. In particular, it allows to effectively separate the $\nu_{\mu}$ and $\bar{\nu}_{\mu}$ signals, and thus to reduce the partial cancellation of their contributions to the $\mathrm{MH}$ asymmetry. The idea was mentioned in 12 . Using the inelasticity will also allow to reduce kinematical smearing effect and degeneracy of parameters. It will lead to a better flavor identification of the $\nu_{\mu}$ events.

The paper is organized as follows. In Sec. II. we describe the $\nu_{\mu}$ events, their detection characteristics, relevant kinematics and cross-sections. In Sec. III possible improvements of the sensitivity to the mass hierarchy and other neutrino parameters due to the inelasticity measurements are discussed. In Sec. IV we compute the three dimensional distributions of events in the reconstructed neutrino energy, $E_{\nu}$, the muon zenith angle, $\theta_{\mu}$, and $y$ variables, which take into account the kinematical smearing. We explore the properties of these 3D distributions and find the corresponding hierarchy asymmetry plots. In Sec. V] we present results of smearing of the distributions over the finite experimental resolutions of observables: the muon and hadron cascade energies and the muon angle. We then compute the total significance of identification of the mass hierarchy, and its dependence on possible accuracy of measurements of the energies and angles (experimental smearing). We estimate how measurements of inelasticity reduce the effect of degeneracy of the mass hierarchy and mass splitting $\Delta m_{32}^{2}$. Sec. VI contains discussion of the results and outlook. 


\section{II. $\nu_{\mu}$ EVENTS AND INELASTICITY}

\section{A. $\nu_{\mu}$ events}

In this paper we concentrate on the $\nu_{\mu}$ events induced by the charged current weak interactions:

$$
\nu_{\mu}+N \rightarrow \mu+h,
$$

where $h$ refers to the hadron system in the final state. Observables associated to the reaction (1) are the energy of the muon $E_{\mu}$, its direction characterized by the zenith and azimuth angles $\theta_{\mu}$ and $\phi_{\mu}$, and the cascade energy (the total energy in hadrons), $E_{h}$.

At the energies we consider, from a few $\mathrm{GeV}$ up to $\approx 30 \mathrm{GeV}$, the cascade direction is not meaningful on an event basis. Indeed, the cascade energy is shared in a variable mixture between light mesons and heavier hadrons, which leads to a highly random and anisotropic Cherenkov photons emission. Contrary to muons, the energy release from cascades is approximately "point-like", given the sparsely instrumented detector arrays under investigation.

The reconstruction of the $\nu_{\mu}$ event consisting of recorded photons (hits) from the combined emission from a vertex shower and a muon track in this case, can be performed well at low energy using prescriptions in 13. The visible cascade energy, the muon track length (i.e. its energy) and incoming muon direction can be extracted. Moreover, the impact of the short scattering length of Cherenkov photons for a dense detector in ice is expected to be rather mild as it will be argued later. As most hits are undelayed, a good reconstruction accuracy of the muon incoming direction as well as a clear separation of the shower and muon signatures are expected.

Thus, the set of observables $\left\{E_{\mu}, \theta_{\mu}, \phi_{\mu}, E_{h}\right\}$, constitutes a rather exhaustive description of the CC $\nu_{\mu}$ interaction. The original neutrino energy is determined through

$$
E_{\nu}=E_{h}+E_{\mu}-m_{N},
$$

where $m_{N}$ is the nucleon mass.

\section{B. Inelasticity and kinematics of the process}

The inelasticity $y$ is defined as

$$
y \equiv \frac{E_{\nu}-E_{\mu}}{E_{\nu}},
$$

Let us consider the angle between the neutrino and the produced muon, $\beta$. The square of the transfer momentum, $q^{2}$, equals

$$
q^{2} \equiv\left(p_{\nu}-p_{\mu}\right)^{2}=-2 E_{\nu}\left(E_{\mu}-\left|\mathbf{p}_{\mu}\right| \cos \beta\right)+m_{\mu}^{2},
$$

so that $Q^{2} \equiv-q^{2}$ equals

$$
Q^{2}=2 E_{\nu} E_{\mu}\left(1-\frac{\left|\mathbf{p}_{\mu}\right|}{E_{\mu}} \cos \beta\right)-m_{\mu}^{2} .
$$

This gives

$$
\cos \beta=\frac{E_{\mu}}{\left|\mathbf{p}_{\mu}\right|}\left(1-\frac{Q^{2}+m_{\mu}^{2}}{2 E_{\nu} E_{\mu}}\right) .
$$

In terms of the Bjorken variable

$$
x \equiv \frac{Q^{2}}{2\left(p_{N} q\right)}=\frac{Q^{2}}{2 m_{N}\left(E_{\nu}-E_{\mu}\right)}
$$

we have

$$
Q^{2}=2 x y m_{N} E_{\nu},
$$

where we used (3). Insertion of this expression into (4) gives

$$
\cos \beta=\frac{E_{\mu}}{\left|\mathbf{p}_{\mu}\right|}\left[1-\frac{2 x y m_{N} E_{\nu}+m_{\mu}^{2}}{2 E_{\nu} E_{\mu}}\right] .
$$

Notice that with decrease of $\mathbf{p}_{\mu}, \cos \beta \rightarrow \pm 1$ when $x \rightarrow$ 0,1 . We can rewrite (5) as

$$
\cos \beta=1-2 x y \zeta\left(E_{\nu}, x, y\right),
$$

where

$$
\zeta\left(E_{\nu}, x, y\right)=\frac{m_{\mu}^{2}+2 m_{N} E_{\nu} x y-2 E_{\nu}\left(E_{\mu}-\left|\mathbf{p}_{\mu}\right|\right)}{4 E_{\nu} x y\left|\mathbf{p}_{\mu}\right|} .
$$

Here $E_{\mu}=E_{\mu}\left(E_{\nu}, y\right)$ and $\mathbf{p}_{\mu}=\mathbf{p}_{\mu}\left(E_{\nu}, y\right)$. If $\mathbf{p}_{\mu} \approx E_{\mu} \gg$ $m_{\mu}$, we obtain neglecting $m_{\mu}$

$$
\zeta\left(E_{\nu}, y\right) \approx \frac{m_{N}}{2 E_{\mu}} .
$$

Let us find the limits in which $c_{\beta} \equiv \cos \beta$ changes. Varying $x$, we obtain for $x=0$ that $c_{\beta} \approx E_{\mu} /\left|\mathbf{p}_{\mu}\right|>1$, so that $c_{\beta}^{\max }=1$. For not very small $\left|\mathbf{p}_{\mu}\right|$ (and we will consider $\left|\mathbf{p}_{\mu}\right|>m_{N}$ ) the minimal value of $c_{\beta}$ corresponds to $x=1$ :

$$
\begin{aligned}
c_{\beta}^{\min } & =\frac{E_{\mu}}{\left|\mathbf{p}_{\mu}\right|}\left[1-\frac{2 m_{N}\left(E_{\nu}-E_{\mu}\right)+m_{\mu}^{2}}{2 E_{\nu} E_{\mu}}\right] \\
& \approx \frac{E_{\mu}}{\left|\mathbf{p}_{\mu}\right|}\left[1-\frac{2 m_{N}}{E_{\mu}}\left(1-\frac{E_{\mu}}{E_{\nu}}\right)\right],
\end{aligned}
$$

or $\sin \beta^{\min } / 2 \sim \sqrt{y m_{N} / E_{\mu}}$.

For a given muon direction, the neutrino direction is determined by the angle $\beta$ and the azimuthal angle $\phi$ with respect to the plane formed by the muon momentum and axis $x$. It is straightforward (see Appendix A) to find the relation between the neutrino zenith angle $\theta_{\nu}$ and the muon zenith angle $\theta_{\mu}$ :

$$
c_{\nu}=c_{\beta} c_{\mu}+s_{\beta} s_{\mu} c_{\phi},
$$

where $c_{\nu} \equiv \cos \theta_{\nu}, c_{\mu} \equiv \cos \theta_{\mu}$ and $c_{\phi} \equiv \cos \phi$.

According to (7) for fixed $\beta$ the maximal and minimal values of $c_{\nu}$ correspond to $c_{\phi}= \pm 1$ and equal

$$
c_{\nu}^{\max }=\cos \left(\theta_{\mu}-\beta\right), \quad c_{\nu}^{\min }=\cos \left(\theta_{\mu}+\beta\right) .
$$




\section{Cross-sections}

In our calculations of Secs. IV and $\mathrm{V}$ we will use the deep inelastic scattering (DIS) cross-section only. We neglect the contributions of the single pion production and quasi-elastic scattering processes. This will lead to conservative estimations of sensitivities, as the reduced momentum transfer translates into smaller angle between the muon and the neutrino directions. In any case the relative importance of these processes becomes negligible above $\approx 5 \mathrm{GeV}$, and below $\approx 5 \mathrm{GeV}$, detector resolutions and effective volumes are strongly limited.

The differential CC cross-sections of the $\nu$ and $\bar{\nu}$ DIS on an isonucleon $\mathrm{N}=\frac{1}{2}(\mathrm{n}+\mathrm{p})$ equal

$$
\begin{aligned}
& \frac{\mathrm{d}^{2} \sigma_{\nu}^{\mathrm{CC}}}{\mathrm{d} x \mathrm{~d} y}\left(E_{\nu}, x, y\right)=\frac{G_{F}^{2} m_{\mathrm{N}} x E_{\nu}}{\pi} \\
& \quad \times\left[(q+s-c)+(1-y)^{2}(\bar{q}-\bar{s}+\bar{c})\right], \\
& \frac{\mathrm{d}^{2} \sigma_{\bar{\nu}}^{\mathrm{CC}}}{\mathrm{d} x \mathrm{~d} y}\left(E_{\nu}, x, y\right)=\frac{G_{F}^{2} m_{\mathrm{N}} x E_{\nu}}{\pi} \\
& \quad \times\left[(\bar{q}-\bar{s}+\bar{c})+(1-y)^{2}(q+s-c)\right],
\end{aligned}
$$

where $q \equiv u+d+s+c, \bar{q} \equiv \bar{u}+\bar{d}+\bar{s}+\bar{c}$ and the quark densities $u=u\left(x, Q^{2}\right)$, etc., are described by the CTEQ5 parton distribution functions in the standard $\overline{\mathrm{MS}}$ scheme [14, valid down to $Q^{2} \approx 1 \mathrm{GeV}^{2}$.

The limits of $x$-integration of the cross sections are in the interval $\left\{x_{\min }, x_{\max }\right\}=\left\{x\left(c_{\beta}=1\right), 1\right\}$, and $y$ integration runs from 0 to $y_{\max }=1-m_{\mu} / E_{\nu}$.

Integrating the cross-sections (9) and 10 over $x$ we obtain

$$
\begin{aligned}
\frac{\mathrm{d} \sigma_{\nu}^{\mathrm{CC}}}{\mathrm{d} y} & =\left[-a_{0}-a_{1}(1-y)^{2}\right] 10^{-38} \mathrm{~cm}^{2} \frac{E_{\nu}}{1 \mathrm{GeV}}, \\
\frac{\mathrm{d} \sigma_{\bar{\nu} \mathrm{CC}}}{\mathrm{d} y} & \left.=\left[-b_{0}-b_{1}(1-y)^{2}\right)\right] 10^{-38} \mathrm{~cm}^{2} \frac{E_{\nu}}{1 \mathrm{GeV}},
\end{aligned}
$$

where $a_{0}=0.72, a_{1}=0.06, b_{0}=0.09$ and $b_{1}=0.69$. Then the normalized inelasticity distributions equal

$$
\begin{aligned}
& p_{\nu} \equiv-\frac{1}{\sigma_{\nu}} \frac{\mathrm{d} \sigma_{\nu}}{\mathrm{d} y} \approx \frac{a_{0}+a_{1}(y-1)^{2}}{a_{0}+a_{1} / 3} \\
& p_{\bar{\nu}} \equiv-\frac{1}{\sigma_{\bar{\nu}}} \frac{\mathrm{d} \sigma_{\bar{\nu}}}{\mathrm{d} y} \approx \frac{b_{0}+b_{1}(y-1)^{2}}{b_{0}+b_{1} / 3}
\end{aligned}
$$

Here, we have dropped the very weak $E_{\nu}$-dependence in the range of interest and considered the limit $m_{\mu} \rightarrow 0$.

\section{Number of events}

The number of neutrino and antineutrino events in the case of NH, $N_{\nu}^{N H}$ and $N_{\bar{\nu}}^{N H}$ in a given $i j-$ bin of the size $\Delta_{i} \cos \theta_{\nu}, \Delta_{j} E_{\nu}$ equals

$$
N_{\nu}^{N H}=\int_{\Delta_{i} \cos \theta_{\nu}} \mathrm{d} \cos \theta_{\nu} \int_{\Delta_{j} E_{\nu}} \mathrm{d} E_{\nu} \rho_{\nu}^{N H}\left(E_{\nu}, \cos \theta_{\nu}\right),
$$

and for $N_{\bar{\nu}}^{N H}$ one needs to substitute $\rho_{\nu}^{N H} \rightarrow \rho_{\bar{\nu}}^{N H}$. Here

$$
\begin{aligned}
& \rho_{\nu}^{N H} \equiv 2 \pi N_{A} n_{\text {ice }} V_{\text {eff }} T \sigma^{\mathrm{CC}} \Phi_{\mu}^{0}\left[P_{\mu \mu}^{N H}+\frac{1}{r} P_{\mathrm{e} \mu}^{N H}\right], \\
& \rho_{\bar{\nu}}^{N H} \equiv 2 \pi N_{A} n_{\text {ice }} V_{\mathrm{eff}} T \bar{\sigma}^{\mathrm{CC}} \bar{\Phi}_{\mu}^{0}\left[\bar{P}_{\mu \mu}^{N H}+\frac{1}{\bar{r}} \bar{P}_{\mathrm{e} \mu}^{N H}\right] .
\end{aligned}
$$

In 13, 14,

$$
r \equiv \frac{\Phi_{\mu}^{0}}{\Phi_{\mathrm{e}}^{0}}, \quad \bar{r} \equiv \frac{\bar{\Phi}_{\mu}^{0}}{\bar{\Phi}_{\mathrm{e}}^{0}}
$$

are the flavor ratios, where $\Phi_{\alpha}^{0}=\Phi_{\alpha}^{0}\left(E_{\nu}, \theta_{\nu}\right)$ are the neutrino fluxes at production; $P_{\alpha \beta}^{N H}$ and $\bar{P}_{\alpha \beta}^{N H}$ are the $\nu_{\alpha} \rightarrow \nu_{\beta}$ oscillation probabilities for neutrinos and antineutrinos. $V_{\text {eff }}\left(E_{\nu}\right)$ is the effective volume of a detector, $\rho_{\text {ice }}$ is the ice density, $N_{A}$ is the Avogadro number, and $T$ is the exposure time.

For the effective mass of the detector we take 9

$$
\rho_{\text {ice }} V_{\text {eff }}\left(E_{\nu}\right)=14.6 \times\left[\log \left(E_{\nu} / \mathrm{GeV}\right)\right]^{1.8} \mathrm{Mt} .
$$

We keep the same effective volume as in 9 in spite of several recent re-evaluations for two reasons: (i) for easier comparison of results with those in [9] and some other publications, and (ii) because the final configuration of the detectors are not yet determined. If the effective volume (which also depends on the criteria of selection of events) is reduced by factor $3-4$, the significance for the same exposure period will be reduced by factor 1.7 2.0 .

Expressions for the inverted mass hierarchy is obtained with substitution $\mathrm{NH} \rightarrow \mathrm{IH}$. Recall that in the $2 \nu$ approximation, when effects of 1-2 mixing and mass splitting are neglected, there are relations between the probabilities for normal and inverted hierarchies

$$
P_{\alpha \beta}^{N H}=\bar{P}_{\alpha \beta}^{I H}, \quad P_{\alpha \beta}^{I H}=\bar{P}_{\alpha \beta}^{N H} .
$$

That is, an inversion of the mass hierarchy is equivalent to switching neutrinos and antineutrinos. In the three neutrino mixing context, the relations (15) are not exact (see, e.g., Fig. 2 in [9]), especially for the core crossing trajectories.

\section{IMPACT OF INELASTICITY DETERMINATION. QUALITATIVE PICTURE}

\section{A. Inelasticity and separation of neutrino and antineutrino signals}

The hierarchy asymmetries in the neutrino and antineutrino channels have opposite signs. In fact, in expressions for the difference of numbers of events for $\mathrm{NH}$ and IH (see [9]) all the terms are proportional to the factors $\left(1-\kappa_{\mu}\right)$ and $\left(1-\kappa_{\mathrm{e}}\right)$, where

$$
\kappa_{\mu} \equiv \frac{\bar{\sigma}^{\mathrm{CC}} \bar{\Phi}_{\mu}^{0}}{\sigma^{\mathrm{CC}} \Phi_{\mu}^{0}}
$$


is the ratio of cross-sections and fluxes of the muon antineutrinos and neutrinos at the production, and $\kappa_{\mathrm{e}}$ is defined similarly. The ratios $\kappa_{\mu}$ and $\kappa_{\mathrm{e}}$ depend on neutrino energy and direction and equal approximately $0.4-0.6$. The $\nu$ and $\bar{\nu}$ contributions to the number of events partially cancel each other. So, in this case the MH determination relies on the non-equal $\nu$ and $\bar{\nu}$ fluxes and cross-sections. The separation of the neutrino and antineutrino signals allows to further reduce the cancellation and therefore to enhance the significance. As follows from Eqs $(11,12$, the average value of $y$ is $50 \%$ larger for $\nu$ than for $\bar{\nu}$. Therefore we can use the inelasticity to separate the $\nu$ and $\bar{\nu}$ signals.

One possible procedure is to determine for each bin (a large number of events will allow to do this) the fraction of neutrino and antineutrino events by fitting its $y$-distribution:

1. Select small enough bins in neutrino energy - zenith angle plane, so that the oscillatory structures due to certain mass hierarchy are not averaged out (the bin size should be eventually optimized). The number of neutrino and antineutrino events in each bin equals

$$
N_{\bar{\nu}}=N \alpha \quad N_{\nu}=N(1-\alpha),
$$

where $\alpha$ is the fraction of antineutrino events and we have omitted the bin indices.

2. Measure the $y$-distribution of these events.

3. Fit the measured distribution with

$$
p_{\nu \bar{\nu}}(y, \alpha)=(1-\alpha) p_{\nu}(y)+\alpha p_{\bar{\nu}}(y),
$$

where $p_{\nu}(y)$ and $p_{\bar{\nu}}(y)$ are given in (11) and 12), thus, determining the fraction $\alpha$.

A possible enhancement of the sensitivity to the hierarchy due to the separation of the $\nu$ and $\bar{\nu}$ signals can be estimated in the following way. In the described procedure there are two independent observables: the total number of events, $N=N_{\nu}+N_{\bar{\nu}}$, and $\alpha$ extracted from the $y$-distribution with the accuracy $\delta \alpha$. In general,

$$
\delta \alpha \approx \frac{\gamma}{\sqrt{N}}
$$

where $\gamma=\gamma(\alpha, N)$. The error $\delta \alpha$ can be estimated using the method of moments. As we have two parameters, $\alpha$ and $\delta \alpha$, to extract, it is sufficient to calculate the first and second $y$-moments of $p_{\nu \bar{\nu}}(y, \alpha)$ given in Eq. (17). Using expressions 11 12, we obtain the average inelasticity $\langle y\rangle:$

$$
\langle y(\alpha)\rangle=\int y p_{\nu \bar{\nu}}(y, \alpha) \mathrm{d} y \approx 0.494-0.174 \alpha .
$$

The mean deviation $\sigma_{\bar{y}}^{2} \equiv\left\langle(\bar{y}-\langle y\rangle)^{2}\right\rangle$ of the average $\bar{y}$ value after $N$ measurements from the true value $\langle y\rangle$ is

$$
\begin{aligned}
\sigma_{\bar{y}}(\alpha, N)^{2} & =\frac{\sigma_{y}(\alpha)^{2}}{N}=\frac{1}{N}\left[\int y^{2} p_{\nu \bar{\nu}}(y, \alpha) \mathrm{d} y-\langle y(\alpha)\rangle^{2}\right] \\
& \approx \frac{1}{N}\left(0.084+0.010 \alpha-0.030 \alpha^{2}\right) .
\end{aligned}
$$

From Eq. 19 and following a measurement of $\bar{y}$, we obtain a measured value $\tilde{\alpha}$. The average value $\delta \alpha$ is given by

$$
\delta \alpha=\sqrt{\left\langle(\tilde{\alpha}-\alpha)^{2}\right\rangle}=\frac{\sqrt{\left\langle(\bar{y}-\langle y\rangle)^{2}\right\rangle}}{0.174}=5.75 \sqrt{\sigma_{\bar{y}}^{2}} .
$$

Substituting $\sigma_{\bar{y}}^{2}$ from Eq. 20, we obtain

$$
\delta \alpha(\alpha, N) \approx \frac{\gamma}{\sqrt{N}} \sqrt{1+0.115 \alpha-0.362 \alpha^{2}}
$$

where $\gamma=1.66$.

If $\alpha$ is not close to 1 (for all practical purpose $\alpha \lesssim 0.5$ ), $\delta \alpha$ weakly depends on $\alpha$ and we can use Eq. (18).

A detailed investigation of $\delta \alpha(\alpha, N)$ by means of the maximum likelihood method confirms the estimate Eq. (22) for large $N$. For $N \lesssim 100$, this method shows a slight improvement with increasing $\alpha$ with respect to the method of moments. It is worthwhile to further explore this approach.

Let us find the errors $\sigma_{\nu}$ and $\sigma_{\bar{\nu}}$ in the determination of $N_{\nu}$ and $N_{\bar{\nu}}$. According to Eq. (16), variations of $N_{\nu}$ can be written as

$$
\delta N_{\nu}=(1-\alpha) \delta N-N \delta \alpha=(1-\alpha) \sqrt{N}-N \delta \alpha .
$$

The variations $\delta N$ and $\delta \alpha$ are independent and therefore they sum up squared:

$$
\sigma_{\nu}^{2}=(1-\alpha)^{2} N+(\delta \alpha)^{2} N^{2}, \quad \sigma_{\bar{\nu}}^{2}=\alpha^{2} N+(\delta \alpha)^{2} N^{2} .
$$

Assuming that the measured quantities $\tilde{N}$ and $\tilde{\alpha}$ are respectively distributed according to Poisson with mean $N$ and Gaussian with mean $\alpha$ and standard deviation $\delta \alpha$, the exact variance calculation of $N_{\nu, \bar{\nu}}(\tilde{\alpha}, \tilde{N})$ leads to the same result as in Eq. 23), provided that $N \gg \gamma^{2}$.

Using (18), we have for $\mathrm{NH}$

$$
\frac{\sigma_{\nu}}{\sqrt{N^{N H}}}=\sqrt{(1-\alpha)^{2}+\gamma^{2}}, \quad \frac{\sigma_{\bar{\nu}}}{\sqrt{N^{N H}}}=\sqrt{\alpha^{2}+\gamma^{2}} .
$$

The hierarchy asymmetries in the neutrino and antineutrino channels can then be written as

$$
S_{\nu}=\frac{N_{\nu}^{I H}-N_{\nu}^{N H}}{\sigma_{\nu}}, \quad S_{\bar{\nu}}=\frac{N_{\bar{\nu}}^{I H}-N_{\bar{\nu}}^{N H}}{\sigma_{\bar{\nu}}} .
$$

Here we assume that NH is the true hierarchy and therefore corresponding number of events is what is measured.

If $S_{\bar{\nu}}$ and $S_{\nu}$ are independent, the total significance equals

$$
S_{\mathrm{tot}}^{\mathrm{sep}}=\sqrt{S_{\nu}^{2}+S_{\bar{\nu}}^{2}}
$$

It can be rewritten using Eqs. 25 and (24) as

$$
S_{\text {tot }}^{\text {sep }}=\frac{1}{\sqrt{N^{N H}}} \sqrt{\frac{\left(N_{\nu}^{I H}-N_{\nu}^{N H}\right)^{2}}{(1-\alpha)^{2}+\gamma^{2}}+\frac{\left(N_{\bar{\nu}}^{I H}-N_{\bar{\nu}}^{N H}\right)^{2}}{\alpha^{2}+\gamma^{2}}} .
$$


For the significance without $\nu-\bar{\nu}$ separation we would have

$$
\left|S_{\mathrm{tot}}\right|=\left|\frac{N_{\nu}^{I H}+N_{\bar{\nu}}^{I H}-N_{\nu}^{N H}-N_{\bar{\nu}}^{N H}}{\sqrt{N^{N H}}}\right| .
$$

Therefore the enhancement factor $R \equiv S_{\text {tot }}^{\text {sep }} /\left|S_{\text {tot }}\right|$ due to separation of the neutrino and antineutrino signals equals

$$
R=\frac{1}{1-\kappa_{\mu} f_{P}} \frac{1}{\sqrt{\alpha^{2}+\gamma^{2}}} \sqrt{\frac{\alpha^{2}+\gamma^{2}}{(1-\alpha)^{2}+\gamma^{2}}+\left(\kappa_{\mu} f_{P}\right)^{2}} .
$$

Here

$$
\kappa_{\mu} f_{P}=-\frac{N_{\bar{\nu}}^{I H}-N_{\bar{\nu}}^{N H}}{N_{\nu}^{I H}-N_{\nu}^{N H}}
$$

and

$$
f_{P} \equiv \frac{\bar{P}_{\mu \mu}^{N H}-\bar{P}_{\mu \mu}^{I H}+\frac{1}{\bar{r}}\left(\bar{P}_{\mathrm{e} \mu}^{N H}-\bar{P}_{\mathrm{e} \mu}^{I H}\right)}{P_{\mu \mu}^{I H}-P_{\mu \mu}^{N H}+\frac{1}{r}\left(P_{\mathrm{e} \mu}^{I H}-P_{\mathrm{e} \mu}^{N H}\right)} .
$$

If $\bar{r}=r$, in the $2 \nu$ approximation we would have $f_{P}=1$.

In Eq. (26) the minus sign in the denominator of the first factor reflects the partial cancellation of the hierarchy asymmetries from the neutrino and antineutrino channels. The second factor describes the reduction of enhancement due to the error in the separation of the neutrino and antineutrino signals. The expression is valid if $\alpha$ is not very close to 0 or 1 .

Notice that the enhancement factor $R$ does not depend explicitly on the number of events. The number of events is mainly encoded in $\kappa_{\mu} f_{P}$ and in $\gamma$. The value of $\alpha$ changes from bin to bin. For $\alpha=0.50(0.32), \kappa_{\mu}=0.4$ and $f_{P}=1$ we obtain $R=1.05$ (1.01).

The enhancement factor is very close to unity. However, a slight improvement on the determination of $\gamma$ leads to a substantial increase of $R$. A $10 \%$ decrease of $\gamma$ leads to $R=1.15$ (1.10) for $\alpha=0.50(0.32)$.

Notice that according to 25 , the ratio

$$
\frac{S_{\bar{\nu}}}{S_{\nu}}=-f_{p} \kappa_{\mu} \frac{\sigma_{\nu}}{\sigma_{\bar{\nu}}}
$$

is negative and $\nu$ and $\bar{\nu}$ asymmetries have opposite signs.

For ideal separation, $\gamma=0$, we would have

$$
R=\frac{1}{1-\kappa_{\mu} f_{P}} \frac{1}{\alpha} \sqrt{\frac{\alpha^{2}}{(1-\alpha)^{2}}+\left(\kappa_{\mu} f_{P}\right)^{2}} .
$$

It gives $R=3.6(3.2)$ for $\alpha=0.50$ (0.32). This number can be considered as the maximal possible enhancement.

Notice that the estimations presented above differ from the estimations in the case in which the numbers of $\nu_{\mu}$ and $\bar{\nu}_{\mu}$ events are measured independently (in our previous consideration these numbers correlate). In the latter, $\sigma_{\nu}=\sqrt{N_{\nu}}, \sigma_{\bar{\nu}}=\sqrt{N_{\bar{\nu}}}$ and the enhancement factor equals

$$
R=\frac{1}{1-\kappa_{\mu} f_{P}} \frac{1}{\sqrt{\alpha}} \sqrt{\frac{\alpha}{1-\alpha}+\left(\kappa_{\mu} f_{P}\right)^{2}} .
$$

If $f_{P}=1$ and $\kappa_{\mu} \approx 0.5$, we obtain $R=2.4$ for $\alpha=0.32$.

The above estimations have been done for a single bin and one should average the enhancement factor over all the bins. Since $R$ depend weakly on $N$, the estimation for $\alpha \sim 0.5$ give good idea about the overall enhancement.

Notice that the weak enhancement factor we obtain is due to the error of the separation parameter, $\delta \alpha$. This is confirmed by exact computations in Sec. V

\section{B. Inelasticity and reconstruction of neutrino direction}

The dominant source of sensitivity loss for the determination of the neutrino mass hierarchy follows from the angular smearing of the oscillograms [9, and in particular, the kinematical smearing due to the angle between the neutrino and muon directions. Indeed, according to (5),

$$
\sin ^{2} \frac{\beta}{2} \approx \frac{Q^{2}}{4 E_{\nu} E_{\mu}} \approx \frac{m_{N} x y}{2 E_{\mu}} .
$$

From this relation with $\langle x\rangle \approx 0.3$ we find that the average angle which characterizes the kinematical smearing is

$$
\langle\beta\rangle \approx \frac{0.75}{\sqrt{E_{\nu} / \mathrm{GeV}}} \sqrt{\frac{y}{1-y}} .
$$

Then for the average values $y_{\nu} \approx 0.5$ and $y_{\bar{\nu}} \approx 0.3$ it equals $\left\langle\beta_{\nu}\right\rangle \approx 0.75 / \sqrt{E_{\nu} / \mathrm{GeV}}$ and $\left\langle\beta_{\bar{\nu}}\right\rangle \approx 0.5 / \sqrt{E_{\nu} / \mathrm{GeV}}$. Using these estimations we find that $\Delta c_{\nu}$ is larger than the region of the same sign hierarchy asymmetry for $E_{\nu}<6 \mathrm{GeV}$.

According to (27), interactions with small $y$ correspond to small scattering angles. Thus, the selection of events with small $y$ reduces the interval of possible values of $\beta$. For instance, for a sample with $y<0.3$, the average inelasticity is about $\langle y\rangle \approx 0.14$. Then according to (28) the average angle between the muon and neutrino incoming directions equals $\langle\beta\rangle \approx 0.13 / \sqrt{E_{\nu} / \mathrm{GeV}}$. The sample however retains about $30 \%$ of neutrino and $55 \%$ of antineutrino events, thus having lower statistics.

At small $y$, the angular reconstruction error of the muon itself is small, as the muon carries most of the neutrino energy and there are less hits from the cascade, which otherwise worsen the reconstruction of the muon direction.

However, at small $y$, the difference of cross-sections of neutrinos and antineutrinos becomes smaller (they are equal at $y=0$ ). Therefore the separation of the neutrino and antineutrino signal becomes difficult, and the cancellation of neutrino and antineutrino signals in the hierarchy asymmetry becomes stronger.

For large $y$, on the other hand, the contribution of $\bar{\nu}$ is strongly suppressed, which eliminates the $\nu-\bar{\nu}$ cancellation. But for events with large $y$ the reconstruction of 
the neutrino direction is very poor. Furthermore, identification of the $\nu_{\mu}$ events becomes difficult (see below).

\section{Inelasticity, systematic errors and degeneracy of parameters}

In 9, the method is mainly based on the differential measurement of the neutrino-induced muon flux from different incoming directions and at various energies, avoiding some sources of systematic uncertainties (especially the correlated ones). The approach adopted in this paper goes a step beyond with the additional sensitivity to the $\nu_{\mu}-\bar{\nu}_{\mu}$ admixture or $y$ providing the method an even stronger immunity to sources of systematic uncertainties.

The degeneracy of the neutrino parameters reduces significantly the sensitivity to the mass hierarchy 9 . The problem may be alleviated, but not avoided, in the future by more precise measurement of neutrino parameters in MINOS, T2K, NOvA and in reactor experiments. The use of inelasticity in analyses will reduce the impact of degeneracies. Indeed, effects of uncertainties, e.g. in $\Delta m_{32}^{2}$ and $\theta_{23}$, are nearly the same for $\nu$ and $\bar{\nu}$, while the $y$-distributions for $\nu$ and $\bar{\nu}$ are different. Therefore measurements of inelasticity will allow to somehow separate effects. A quantitative study of the corresponding improvements will be given in Sec. V

\section{Inelasticity and identification of $\nu_{\mu}$ events}

As discussed in 9], tau neutrinos contaminate the $\nu_{\mu}$ sample by about $5 \%$ contribution. Oscillation effect on this contribution differs from the one on the true $\nu_{\mu}$ events. This leads to a kind of additional smearing, which cannot be neglected. The inelasticity observable enables us to further suppress the number of $\nu_{\tau} \rightarrow \mu$ events in a sample because of the specific vertex kinematics of tau neutrino interactions: Rather large showers are produced and the angle between the muon and tau neutrino is large, as the muon is sharing energy with two other neutrinos. Therefore this class of events has in average rather large effective $y$, and restricting an analysis to small $y$ will allow to disentangle at least partly the $\nu_{\tau}$ contribution. Quantitative analysis of this suppression is beyond the scope this paper.

For large $y$, due to the low energy of muon, the probability of misidentification of the $\nu_{\mu}$ events with the $\mathrm{CC}$ $\nu_{\mathrm{e}, \tau}$ events as well as the $\mathrm{NC}$ events of all neutrino species becomes large. Indeed, there can be confusion between the charged pion and muons as they both have a long decay length $\left(\lambda_{\pi^{ \pm}} \approx 56 \mathrm{~m}\right.$ at $\left.1 \mathrm{GeV}\right)$ and propagate with low energy loss rate (the dominant ionization energy loss limits their range to $\lesssim 5 \mathrm{~m}$ per $\mathrm{GeV}$ ). However, the energy distributions of muon and pions strongly differ: the simulation with GENIE [15] of $10 \mathrm{GeV} \nu_{\mu}$ interactions shows that the most probable $E_{\pi^{ \pm}}$is of the order of a few 100 's $\mathrm{MeV}$, so that $E_{\pi^{ \pm}} \approx 1 \mathrm{GeV}$ is already unlikely high. The reaction favors events with higher $\pi$ multiplicity rather than events with higher $\pi$ energies.

\section{3D - DISTRIBUTIONS AND OSCILLOGRAMS}

As we saw in the previous section the separation of the neutrino and antineutrino signals requires measurements of the $y$-distribution in a wide range of $y$, and especially for large $y$, where the difference of the neutrino and antineutrino cross-section is maximal. On the other hand, good reconstruction of the neutrino directions requires selection of events with small $y$. In a sense, improvements of the sensitivity due to $\nu-\bar{\nu}$ separation and narrowing the angular distribution are incompatible. Small $y$ are preferred also for the identification of the $\nu_{\mu}$ events and the disentanglement of the $\nu_{\mu}$ from $\nu_{e, \tau}$ events. Therefore, one expects that the best sensitivity to the neutrino mass hierarchy is for the intermediate range of $y$. Here the interplay of different effects occurs, which requires a combined description using the differential characteristics in $y$ and also in $x$ since the angle depends on $x$ too.

\section{A. Densities of events. Oscillograms for different $y$}

The density of the $\nu_{\mu}$ events as function of $E_{\nu}, c_{\mu}, y$ equals

$n_{\nu}^{N H}\left(E_{\nu}, c_{\mu}, y\right)=\frac{1}{2 \pi} \int_{c_{\beta}^{\min }}^{1} \mathrm{~d} c_{\beta} \int_{0}^{2 \pi} \mathrm{d} \phi \frac{\mathrm{d}^{2} \sigma_{\nu}^{\mathrm{CC}}}{\mathrm{d} c_{\beta} \mathrm{d} y} \frac{\rho_{\nu}^{N H}\left(E_{\nu}, c_{\nu}\right)}{\sigma_{\nu}^{\mathrm{CC}}\left(E_{\nu}\right)}$,

where $\rho_{\nu}^{N H}$ is defined in 13$) ; c_{\nu}$ is given in (7), and the lower limit of integration, $c_{\beta}^{\mathrm{min}}$, is defined in $(\overrightarrow{6})$. Similar expressions can be written for antineutrinos and for the IH case.

Using the relation Eq. (7), we change the integration variables, $d \phi \rightarrow d c_{\nu}$ :

$$
\begin{array}{r}
n_{\nu}^{N H}\left(E_{\nu}, c_{\mu}, y\right)=\frac{1}{\pi} \int_{c_{\beta}^{\min }}^{1} \mathrm{~d} c_{\beta} \frac{\mathrm{d}^{2} \sigma_{\nu}^{\mathrm{CC}}}{\mathrm{d} c_{\beta} \mathrm{d} y}\left(x\left(c_{\beta}\right), y\right) \\
\quad \times \int_{c_{\nu}^{\min }}^{c_{\nu}^{\max }} \mathrm{d} c_{\nu} \frac{1}{\sqrt{h\left(c_{\mu}, c_{\beta}, c_{\nu}\right)}} \frac{\rho_{\nu}^{N H}\left(E_{\nu}, c_{\nu}\right)}{\sigma_{\nu}^{\mathrm{CC}}\left(E_{\nu}\right)} .
\end{array}
$$

Here

$$
h \equiv\left(s_{\mu} s_{\beta}\right)^{2}-\left(c_{\nu}-c_{\mu} c_{\beta}\right)^{2},
$$

and $1 / \sqrt{h}$, is essentially the Jacobian of transition to new variables according to (7). The limits of integration $c_{\nu}^{\max }$ and $c_{\nu}^{\min }$ will be specified later. Notice that appearance of an additional factor 2 in the expression 29 is due to twofold ambiguity at the transition from $\phi$ to $c_{\nu}$.

Let us make another change of the integration variable: $c_{\beta} \rightarrow x$. Using the equality

$$
\frac{\mathrm{d}^{2} \sigma_{\nu}^{\mathrm{CC}}}{\mathrm{d} c_{\beta} \mathrm{d} y} \mathrm{~d} c_{\beta}=\frac{\mathrm{d}^{2} \sigma_{\nu}^{\mathrm{CC}}}{\mathrm{d} x \mathrm{~d} y} \mathrm{~d} x
$$


we obtain from 29

$$
\begin{aligned}
n_{\nu}^{N H}\left(E_{\nu}, c_{\mu}, y\right) & =\frac{1}{\pi} \int_{x_{\min }}^{x_{\max }} \mathrm{d} x \int_{c_{\nu}^{\min }}^{c_{\nu}^{\max }} \mathrm{d} c_{\nu} \frac{d^{2} \sigma_{\nu}^{\mathrm{CC}}}{d x d y} \\
\times & \frac{1}{\sqrt{h\left(c_{\mu}, c_{\beta}, c_{\nu}\right)}} \frac{\rho_{\nu}^{N H}\left(E_{\nu}, c_{\nu}\right)}{\sigma_{\nu}^{\mathrm{CC}}\left(E_{\nu}\right)} .
\end{aligned}
$$

Here $x_{\text {min,max }}$ correspond to the values $c_{\beta}\left(E_{\nu}, x, y\right)= \pm 1$. In turn, the limits of integration over $c_{\nu}$ correspond to $h=0$, i.e. to the borders of the interval of the positivity condition: $h \geq 0$. Indeed, the expression for $h$ can be rewritten as

$$
h=-\left[c_{\nu}-\cos \left(\theta_{\mu}-\beta\right)\right] \times\left[c_{\nu}-\cos \left(\theta_{\mu}+\beta\right)\right],
$$

where $\beta=\beta\left(x, y, E_{\nu}\right)$ is determined in Eq. (4). Then the limits $c_{\nu}^{\max }=\cos \left(\theta_{\mu}-\beta\right)$ and $c_{\nu}^{\min }=\cos \left(\theta_{\mu}+\beta\right)$ follow immediately.

Changing the order of integrations over $x$ and $c_{\beta}$, we obtain

$$
\begin{aligned}
n_{\nu}^{\mathrm{NH}}\left(E_{\nu}, c_{\mu}, y\right)= & \frac{1}{\pi} \int_{\left|\theta_{\mu}-\theta_{\nu}\right| \leq \beta_{0}} \mathrm{~d} c_{\nu} \rho_{\nu}^{\mathrm{NH}}\left(E_{\nu}, c_{\nu}\right) \\
\times & g_{\nu}\left(E_{\nu}, y, c_{\nu}, c_{\mu}\right),
\end{aligned}
$$

where

$$
\begin{aligned}
g_{\nu}\left(E_{\nu}, y, c_{\nu}, c_{\mu}\right) & \equiv \frac{1}{\sigma_{\nu}^{\mathrm{CC}}\left(E_{\nu}\right)} \int_{x^{-}}^{x^{+}} \mathrm{d} x \frac{\mathrm{d}^{2} \sigma_{\nu}^{\mathrm{CC}}\left(E_{\nu}, x, y\right)}{\mathrm{d} x \mathrm{~d} y} \\
\times & \frac{1}{\sqrt{s_{\beta}^{2} s_{\mu}^{2}-\left(c_{\nu}-c_{\beta} c_{\mu}\right)^{2}}}
\end{aligned}
$$

Here $s_{\beta}$ and $c_{\beta}$ are functions of $E_{\nu}, x$ and $y$. The function $g_{\nu}$ does not depend on the mass hierarchy and essentially play the role of the kinematic smearing function.

Writing similar expressions for IH and $\bar{\nu}$, we obtain the densities of the events for $\mathrm{NH}$ and $\mathrm{IH}$ :

$$
\begin{aligned}
& n^{\mathrm{NH}, \mathrm{IH}}\left(E_{\nu}, c_{\mu}, y\right)= n_{\nu}^{\mathrm{NH}, \mathrm{IH}}\left(E_{\nu}, c_{\mu}, y\right)+n_{\bar{\nu}}^{\mathrm{NH}, \mathrm{IH}}\left(E_{\nu}, c_{\mu}, y\right) \\
&=\frac{1}{\pi} \int_{\left|\theta_{\mu}-\theta_{\nu}\right| \leq \beta_{0}} \mathrm{~d} c_{\nu}\left[\rho_{\nu}^{\mathrm{NH}, \mathrm{IH}}\left(E_{\nu}, c_{\nu}\right) g_{\nu}\left(E_{\nu}, y, c_{\nu}, c_{\mu}\right)\right. \\
&\left.+\rho_{\bar{\nu}}^{\mathrm{NH}, \mathrm{IH}}\left(E_{\nu}, c_{\nu}\right) g_{\bar{\nu}}\left(E_{\nu}, y, c_{\nu}, c_{\mu}\right)\right] .
\end{aligned}
$$

Introducing $\rho^{\mathrm{NH}} \equiv \rho_{\nu}^{\mathrm{NH}}+\rho_{\bar{\nu}}^{\mathrm{NH}}$, we can rewrite the expression in 32 as

$$
\begin{aligned}
& n^{\mathrm{NH}}\left(E_{\nu}, c_{\mu}, y\right)= \\
& \quad \frac{1}{\pi} \int_{\left|\theta_{\mu}-\theta_{\nu}\right| \leq \beta_{0}} \mathrm{~d} c_{\nu} \rho^{\mathrm{NH}}\left(E_{\nu}, c_{\nu}\right) G\left(E_{\nu}, y, c_{\nu}, c_{\mu}\right),
\end{aligned}
$$

where

$$
G\left(E_{\nu}, y, c_{\nu}, c_{\mu}\right) \equiv g_{\nu} \frac{\rho_{\nu}^{\mathrm{NH}}}{\rho^{\mathrm{NH}}}+g_{\bar{\nu}} \frac{\rho_{\bar{\nu}}^{\mathrm{NH}}}{\rho^{\mathrm{NH}}} .
$$

The function $G$ can be immediately compared with the Gaussian smearing function, which was used in 9] embedding both kinematic and experimental resolution effects.
Let us find the limits of integration over $x$ in Eq. 31. According to Eq. (5)

$$
x\left(c_{\beta}\right)=\frac{2 E_{\nu}\left(E_{\mu}-\left|\mathbf{p}_{\mu}\right| c_{\beta}\right)-m_{\mu}^{2}}{2 m_{N} E_{\nu} y},
$$

which imposes the lower and upper bounds to $x$ :

$$
x^{ \pm}=x\left(\cos \left(\theta_{\nu} \pm \theta_{\mu}\right)\right) .
$$

For a given $\theta_{\nu}$ and $\theta_{\mu}$, the minimal angle between the muon and the neutrino is $\beta=\left|\theta_{\mu}-\theta_{\nu}\right|$. The maximal angle $\beta$ is given by $c_{\beta, \max }=\cos \left(\theta_{\nu}+\theta_{\mu}\right)$.

The integration over $c_{\nu}$ in Eq. (30) runs from $\cos \left(\theta_{\mu}+\right.$ $\left.\beta_{0}\right)$ to $\cos \left(\theta_{\mu}-\beta_{0}\right)$, where $c_{\beta_{0}}=c_{\beta}\left(E_{\nu}, x=1, y\right)$ and $\theta_{\mu} \pm \beta_{0}$ is restricted by the interval $0-\pi$.

\section{B. Kinematical smearing function}

According to Eq. (30), the functions $g_{\nu, \bar{\nu}}\left(E_{\nu}, y, c_{\nu}, c_{\mu}\right)$ in (31) can be considered as the smearing functions over the neutrino angle. Fig. 1 shows dependence of $g_{\nu}$ and $g_{\bar{\nu}}$ on $c_{\mu}$ for several values of $c_{\nu}$ and $y$.

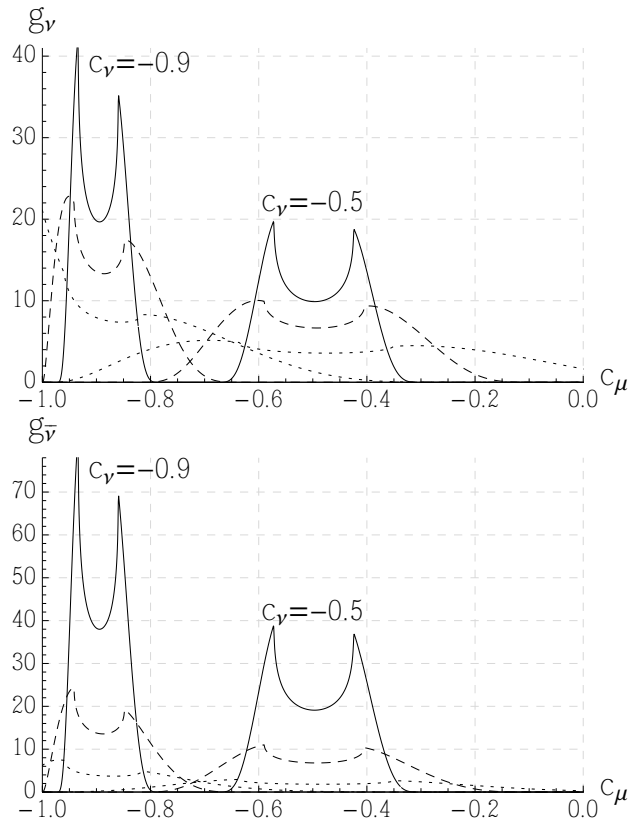

FIG. 1: The angular smearing functions for neutrinos (upper panel) and antineutrinos (bottom panel) for $E_{\nu}=10 \mathrm{GeV}$ and different values of $c_{\nu}$ (numbers at the curves). Solid, dashed and dotted curves are respectively for $y=0.2, y=0.5$ and $y=0.8$.

The smearing functions differ from the Gaussian function assumed in 9]. They have two peaks with a local minimum in between; there are no exponential tails; the central parts are at $c_{\mu} \approx c_{\nu}$. The asymmetry of the peaks becomes stronger with $c_{\mu}$ approaching \pm 1 ; the width of the functions increases with $y$. The functions $g$ are similar for neutrinos and antineutrinos. As expected, for 
antineutrinos the overall normalization decreases with increase of $y$, whereas for neutrinos normalization changes weakly.

The properties of $g_{\nu, \bar{\nu}}$ can be readily understood from the expression for $h$. Indeed, $g_{\nu, \bar{\nu}}$ have inverted (and also smoothed) shapes with respect to that of $\sqrt{h}$. In particular, peaks of $g_{\nu, \bar{\nu}}$ correspond to zeros of $h$, the minima of $g_{\nu, \bar{\nu}}$ correspond to the maxima of $h$, etc..

The function $h$ can be rewritten as

$$
h=s_{\beta}^{2}-\left(c_{\nu}^{2}+c_{\mu}^{2}\right)+2 c_{\nu} c_{\mu} c_{\beta},
$$

which is obviously symmetric with respect to the interchange

$$
c_{\nu} \leftrightarrow c_{\mu}
$$

As a consequence, $g_{\nu, \bar{\nu}}$ also obeys this symmetry. Introducing

$$
r \equiv 2 \zeta x y=2 \sin ^{2} \frac{\beta}{2},
$$

we can present $h$ as

$$
h=2 r\left(1-c_{\nu} c_{\mu}\right)-r^{2}-\left(c_{\nu}-c_{\mu}\right)^{2} .
$$

Then defining the difference $\Delta \equiv c_{\nu}-c_{\mu}$, we have

$$
h(\Delta)=s_{\mu}^{2}\left(2 r-r^{2}\right)-\left(\Delta+r c_{\mu}\right)^{2} .
$$

So, $h$, as function of $\Delta$, is an inverted parabola with its maximum shifted to $\Delta=-r c_{\mu}$. In agreement with Fig. 1. $h(\Delta)$ is not symmetric with respect to $\Delta=0$ or $c_{\nu}=c_{\mu}$, and the minimum of $g_{\nu, \bar{\nu}}$ is shifted with respect to $c_{\mu}=c_{\nu}$. This also leads to difference of heights of peaks.

Zeroes of $h$ are at

$$
\Delta=-r c_{\mu} \pm \sqrt{s_{\mu}^{2}\left(2 r-r^{2}\right)} .
$$

According to 8 in terms of angles the zeros of $h$ are given by $\cos \theta_{\nu}=\cos \left(\theta_{\mu} \pm \beta\right)$. So, the width of the smearing function increases with $\beta$. In turn, according to (28), $\beta \propto \sqrt{y}$, and consequently, the width increases with $y$, as we mentioned before.

It is easy to understand the appearance of peaks in $g_{\nu, \bar{\nu}}\left(c_{\nu}\right)$ at the borders of allowed interval using the following graphical representation. The neutrino vector is on the surface of the cone with angle $\beta$ and axis along the muon momentum. With change of $\phi$, the neutrino vector moves on the surface of the cone. The maximum and minimum of $c_{\nu}$ given by (8) correspond to the neutrino vector situated in the plane formed by the muon vector and the axis $z$ and the neutrino vector is moving perpendicularly to this plane. Therefore around these positions the $z$-projection of the neutrino vector does not change appreciably, and so the integration over $\phi$ leads to bigger contribution.

\section{Oscillograms for different values of $y$}

We will use the general formulas obtained in the previous sections to compute the oscillograms and asymmetry distributions for different values of $y$. The functions $\rho_{\nu, \bar{\nu}}^{\mathrm{NH}, \mathrm{IH}}\left(E_{\nu}, c_{\nu}\right)$ are taken from $[9$.

Fig. 2 shows the $E_{\nu}-\cos \theta_{\mu}$ binned distribution of the hierarchy asymmetry with the inelasticity ( $y$-dependence) and kinematical smearing taken into account. Different panels in this figure correspond to different $y$-intervals $\left\{y_{\min }, y_{\max }\right\}$. The asymmetry in these intervals has been computed in the following way. We first used very small $y$-bins $\Delta y \ll\left(y_{\max }-y_{\min }\right)$. We computed the asymmetry in each of these small bins $S_{k}=S\left(y_{k}, E_{\nu}, \cos \theta_{\mu}\right)$ and then the total asymmetry in the interval $\left\{y_{\min }, y_{\max }\right\}$ as $S_{\text {tot }}=\sqrt{\sum S_{k}^{2}}$ (the sum runs over all small $y$-bins in the interval $\left.\left\{y_{\min }, y_{\max }\right\}\right)$, and the sign is the same as for dominant contribution. In practice the summation over small bins was substituted by integration:

$S\left(y_{\max }, y_{\min }, E_{\nu}, \cos \theta_{\mu}\right)=\left[\int_{y_{\min }}^{y_{\max }} \mathrm{d} y \frac{\left(n^{\mathrm{IH}}-n^{\mathrm{NH}}\right)^{2}}{n^{\mathrm{NH}}}\right]^{1 / 2}$.

Here $n^{\mathrm{NH}, \mathrm{IH}}$ is the number of events in the bin $\Delta E_{\nu} \Delta \cos \theta_{\mu}$ given in 32 .

The first panel of Fig. 2 corresponds to $\left\{y_{\min }, y_{\max }\right\}=$ $\{0,1\}$, the others - to various intervals with $y_{\max }-y_{\min }=$ 0.2 . The first panel is the sum of contributions described in other panels. As we see the biggest contribution comes from the intermediate region $y \in\{0.3,0.7\}$. Indeed, at small $y$ the hierarchy asymmetry is suppressed due to strong cancellation of the nearly equal contributions from neutrinos and antineutrinos (recall that at $y \sim 0$ the $\nu$ and $\bar{\nu}$ cross-sections become equal). At large $y$, the asymmetry is suppressed due to strong smearing over the angle between muon and neutrino. With the increase of $y$, the region of strong asymmetry first shifts smaller $E_{\nu}$ and larger $\cos \theta_{\mu}$, and then move to larger $E_{\nu}$ and $\cos \theta_{\mu}=-1$. The region expands in horizontal $\left(\cos \theta_{\mu}\right)$ direction for small $y$.

The total significance (given by integration over the first panel with $0 \leq y \leq 1$ ) equals

$$
\left|S_{\text {tot }}\right|=\left[\int \mathrm{d} c_{\mu} \int \mathrm{d} E_{\nu} \int_{0}^{1} \mathrm{~d} y \frac{\left(n^{\mathrm{IH}}-n^{\mathrm{NH}}\right)^{2}}{n^{\mathrm{NH}}}\right]^{1 / 2} .
$$

For exposure $T=1$ year, this leads to $\left|S_{\text {tot }}\right|=8.43$.

If the $y$-dependence is not used, the densities of events should be integrated over $y$ before computing $S$. This gives

$$
\left|S_{\text {tot }}^{\text {int }}\right|=\left[\int \mathrm{d} c_{\mu} \int \mathrm{d} E_{\nu} \frac{\left(\int_{0}^{1} \mathrm{~d} y\left(n^{\mathrm{IH}}-n^{\mathrm{NH}}\right)\right)^{2}}{\int_{0}^{1} \mathrm{~d} y n^{\mathrm{NH}}}\right]^{1 / 2} .
$$

For 1 year exposure we obtain from $34\left|S_{\text {tot }}^{\text {int }}\right|=7.11$, which is about $15 \%$ smaller than in the case when $y$ distribution is used according to Eq. (33). 

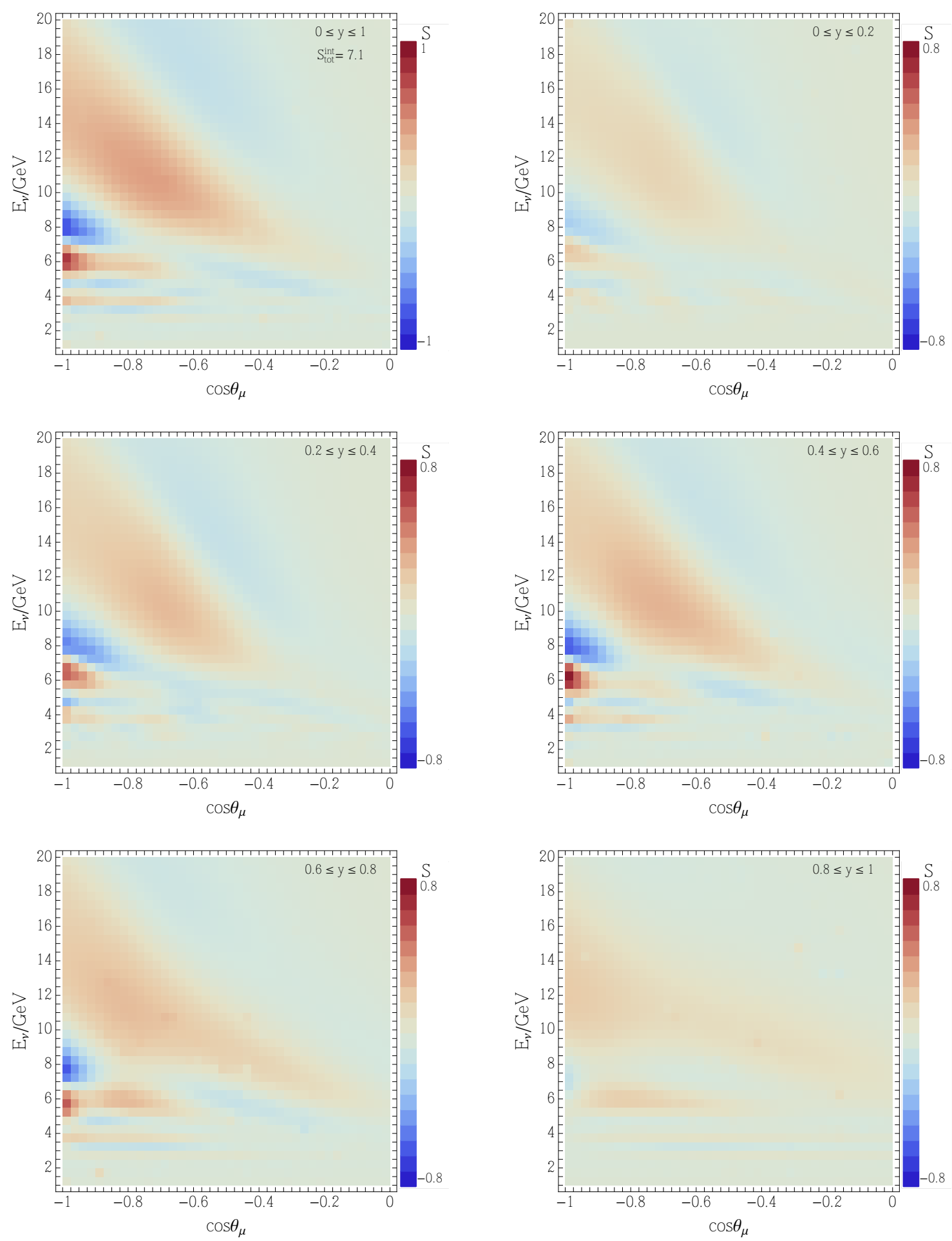

FIG. 2: The hierarchy asymmetry distributions after the kinematical smearing for various inelasticity ranges and for 1 year of exposure.

The following comments are in order:

(i) The kinematical smearing strongly reduces the total significance: for the ideal reconstruction of the neutrino energy and direction we would obtain $\left|S_{\text {tot }}^{\nu+\bar{\nu}}\right|=23.7$ during 1 year even without $y$-information. This number can be considered as maximal achievable significance. It should be compared with $\left|S_{\text {tot }}^{\text {int }}\right|=7.11$ obtained from (34). Note that $\left|S_{\text {tot }}\right|=8.43$ can be obtained with 
an ideal detector having perfect resolutions (see Sec. V).

(ii) The increase of significance by about $15 \%$ with $y$-distribution is better than the one predicted from our qualitative discussion in Sec. III] using $\gamma$ derived from the method of moments. This is probably related to the fact that the characteristics of the $y$-distribution are more fully exploited.

Note also that in our treatment the cross-sections have been restricted to the DIS approximation, thus exhibit cutoffs at small $y$, a region of good angular resolution. Therefore a more complete description of the cross sections will recover the events in the small $y$-region, further enhancing the significance.

\section{SIGNIFICANCE OF DETERMINATION OF MASS HIERARCHY WITH INELASTICITY}

In the previous section we have taken into account the kinematical smearing - the integration over the angle between the neutrino and muon, $\beta$. Besides this, one should perform the experimental smearing over the observables: the energy of muon and cascade as well as the direction of muon due to finite experimental energy and angular resolutions.

\section{A. Experimental resolution functions}

We present here the significance of the identification of the mass hierarchy, considering various scenarios for the widths $\sigma_{\mu, h}\left(E_{\nu}, y\right)$ and $\sigma_{\psi}\left(E_{\nu}, y\right)$ of the energy and angular resolution functions. We use the notation $\tilde{x}$ for the reconstructed value of the observable $x$.

\section{Energy resolution}

We assume the Gaussian energy resolution functions of the cascade and muon with widths $\sigma_{h}$ and $\sigma_{\mu}$ correspondingly. Then the neutrino energy resolution is itself the Gaussian function (sum of two normal distributions):

$$
g_{E_{\nu}}\left(\tilde{E}_{\nu}, E_{\nu}\right)=\frac{1}{\sqrt{2 \pi} \sigma_{E_{\nu}}} \exp \left[-\frac{1}{2} \frac{\left(\tilde{E}_{\nu}-E_{\nu}\right)^{2}}{\sigma_{\nu}^{2}}\right]
$$

with width

$$
\sigma_{\nu}(y)=\sqrt{\sigma_{\mu}^{2}+\sigma_{h}^{2}}
$$

which depends on $y$.

We consider two cases for the energy resolution of muons and cascades:

(i) $\sigma_{\mu, h}=b E_{\mu, h}$, which gives $\sigma_{\mu}=b E_{\mu} \approx b(1-y) E_{\nu}$ and $\sigma_{h}=b E_{h} \approx b y E_{\nu}$, so that

$$
\sigma_{\nu}(y)=b E_{\nu} \sqrt{1-2 y+2 y^{2}} ;
$$

(ii) $\sigma_{\mu, h}=\sqrt{b E_{\mu, h}}$, then

$$
\sigma_{\nu}=\sqrt{b E_{\nu}}
$$

which has the same form as $\sigma_{\mu, h}$. We make here a reasonable simplification that $b$ is the same for cascade and muon. In fact, this is true only if we assume that most of the energy of the cascade is visible like for a muon. More likely, it has a bit smaller Cherenkov photon yield per $\mathrm{GeV}$ and subject to greater event by event fluctuations.

In the case $(i)$, we use $b=0.3$ in order to compare with the results from 9 . In the case $(i i)$, we take $b$ in the range $0.35 \leq b \leq 0.7$, which is derived from an estimated number of detected photons $n_{\text {hit }} / \mathrm{GeV} \approx 1-$ 3 [16. Eq. (26) in [16] predicts $n_{\text {hit }} / \mathrm{GeV} \approx 1.5$ for a mean distance of about $10 \mathrm{~m}$ between the Cherenkov light emitter and an optical module of IceCube type. The range is extended in the mentioned limits, because the precise topology and technology (for instance, the photodetection efficiency and area of the optical modules) of a dense array are not yet precisely known.

We then obtain the energy resolution given by the statistical uncertainty of the number of expected hits:

$$
\begin{aligned}
\sigma_{\mu, h} & =\frac{\delta\left(E_{\mu, h} n_{\mathrm{hit}} / \mathrm{GeV}\right)}{n_{\mathrm{hit}} / \mathrm{GeV}} \\
& \approx \sqrt{\{0.35,1\} E_{\mu, h}} \approx\{0.6,1\} \sqrt{ } E_{\mu, h} .
\end{aligned}
$$

\section{Inelasticity resolution function}

The inelasticity distribution $g_{y}\left(E_{\nu}, y\right)$ can be derived straightly from $E_{\mu, h}$ distributions, $g_{\mu, h}\left(\tilde{E}_{\mu, h}, E_{\mu, h}\right)$, described above. We show in the Appendix B that it is nearly Gaussian in most cases of interests. It deviates from Gaussian, showing enhanced tails, when $E_{\mu}$ and $E_{h}$ are both small. In our computations we use the Gaussian function with width

$$
\sigma_{y}=\frac{1}{\sqrt{ } 2}\left(\frac{E_{h}+\sigma_{h}}{E_{h}+\sigma_{h}+E_{\mu}-\sigma_{\mu}}-y\right) .
$$

Notice that we could perform smearing using immediately $E_{\mu}$ and $E_{h}$ without introducing $y$, and if needed, introduce $\tilde{y}$ after smearing.

\section{Angular resolution}

The angle $\psi$ between the true and the reconstructed muon directions is described by the normalized distribution

$$
g_{\psi}=\frac{2 \psi}{\sigma_{\psi}^{2}} \exp \left(-\frac{\psi^{2}}{\sigma_{\psi}^{2}}\right)
$$

which is derived from the $2 \mathrm{D}$ Gaussian distribution. The interval $\psi \leq \sigma_{\psi}$ encloses $63 \%$, and $g_{\psi}$ peaks at $\sigma_{\psi} / \sqrt{ } 2$. 
The width $\sigma_{\psi}$ is generically a function of $E_{\mu}$, which has the form [13]

$$
\sigma_{\psi}=\psi_{0} \sqrt{\frac{m_{N}}{E_{\mu}}} .
$$

Here $\psi_{0}$ depends on the detector medium (ice, water) and its topology. The IceCube detector is sparsely instrumenting a medium of relatively short scattering length. Therefore a large number of photons will not travel on a straight path between the Cherenkov light emission point and the detection location. On the contrary in ANTARES (and similarly in ORCA), the photons are detected un-delayed. This is the main reason why IceCube has worser angular resolution than the ANTARES detector. Therefore, we consider $\psi_{0}$ values in a range reflecting common angular resolutions achieved in water and by a sparse array in ice. Note, however, that one reasonably expects a substantially improved angular resolution in ice with a smaller and denser array (PINGU), i.e. a global reduction of scale: in this case the short scattering length will be of relative importance and many Cherenkov photons will reach the optical modules closest from their emission point rather undelayed.

The angular resolution of an event with 60 hits is about $5^{\circ}$ in IceCube and better than $2^{\circ}\left(n_{\text {hit }} / \mathrm{GeV} \approx 1.5\right)$ in ANTARES. Therefore $15^{\circ} \lesssim \psi_{0} \lesssim 30^{\circ}$. The range $1 \leq$ $n_{\text {hit }} / \mathrm{GeV} \leq 3$ leads to $8.5^{\circ} \lesssim \psi_{0} \lesssim 40^{\circ}$.

The smearing function for the zenith angle of muon, $g\left(c_{\mu}, \tilde{c}_{\mu}\right)$, can be computed using the smearing function for $\psi(38)$ as

$$
g\left(c_{\mu}, \tilde{c}_{\mu}\right)=\frac{1}{\pi} \int_{\left|\theta_{\mu}-\tilde{\theta}_{\mu}\right|}^{\pi} \frac{g_{\psi}(\psi) \mathrm{d} \psi}{\sqrt{\tilde{s}_{\mu}^{2} s_{\psi}^{2}-\left(\tilde{c}_{\mu} c_{\psi}-c_{\mu}\right)^{2}}} .
$$

The denominator here appears similarly to that at the variable change $\phi \rightarrow c_{\nu}$ performed in Eq. 29). The function $g\left(c_{\mu}, \tilde{c}_{\mu}\right)$ is normalized, which follows from normalization of $g_{\psi}$.

Fig. 3 shows the angular smearing function $g\left(c_{\mu}, \tilde{c}_{\mu}\right)$ for $\psi_{0}=15^{\circ}$ and several values of $\tilde{c}_{\mu}$. Notice that $g_{\psi}\left(c_{\mu}, \tilde{c}_{\mu}\right) \tilde{s}_{\mu}$ is not symmetric and it increasingly deviates from the normal distribution, when approaching $\theta_{\mu}=180^{\circ}$.

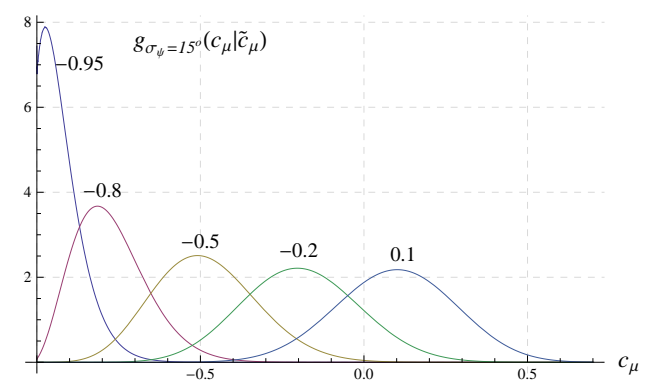

FIG. 3: Angular smearing function in $\cos \theta_{\mu}$ for $\sigma_{\psi}=15^{\circ}$ and several values of $\tilde{c}_{\mu}$.

\section{B. Distributions with experimental smearing}

We calculate the distribution of events smeared over the experimental resolution functions. We convert the 3 -D distributions in the parameter space $E_{\nu}-y-c_{\mu}$ into observed parameters space $\tilde{\theta}_{\mu}, \tilde{E}_{\nu}, \tilde{y}$ convoluting $n$ with the resolution distributions for $E_{\nu}, y$ and $\psi$ :

$$
\hat{n}_{\nu, \bar{\nu}}^{\mathrm{IH}, \mathrm{NH}}\left(\tilde{\theta}_{\mu}, \tilde{E}_{\nu}, \tilde{y}\right)=n_{\nu, \bar{\nu}}^{\mathrm{IH}, \mathrm{NH}}\left(\theta_{\mu}, E_{\nu}, y\right) *\left(g_{\psi} g_{y} g_{\nu}\right) .
$$

The convolution is performed sequentially in order indicated in the last brackets. The smearing functions are taken according to Eqs. (35), (39) and (37) (the width of Gaussian function for $g_{y}$ ). Values of $n_{\nu, \bar{\nu}}^{\mathrm{IH}, \mathrm{NH}}$ outside region $1<E_{\nu} / \mathrm{GeV}<20$ and for $\theta_{\mu}<90^{\circ}$ are set to the values taken at the boundaries.

Integrating over the bins, we obtain the binned oscillogram:

$$
N_{i j k}\left(\tilde{c}_{\mu i}, \tilde{E}_{\nu j}, \tilde{y}_{k}\right)=\int_{\operatorname{bin}(\mathrm{ijk})} \mathrm{d} \tilde{c}_{\mu} \mathrm{d} \tilde{E}_{\nu} \mathrm{d} \tilde{y} \hat{n}_{\nu, \bar{\nu}}^{\mathrm{HH}, \mathrm{NH}}\left(\tilde{\theta}_{\mu}, \tilde{E}_{\nu}, \tilde{y}\right) .
$$

The smeared distributions in the plane $E_{\nu}-\cos \theta_{\mu}$ for different intervals of $y$ and different resolutions are shown in Figs. 4, 5, 6

In comparison with Fig. 2, the overall scale of asymmetries is reduced by factor of $\sim 2$, which quantifies the effect of experimental smearing. Position and shape of the regions of strong asymmetry follow to a large extent those in Fig. 2,

\section{Estimations of the total significance}

Total significances with the experimental smearing are calculated using Eqs. (33 34) with replacement $n \rightarrow$ $\hat{n}$. Table I presents the total significance after one year of exposure for several experimental resolution scenarii, including the one with only kinematical smearing.

For comparison we also compute the significances obtained immediately from the neutrino oscillograms, which corresponds to exact reconstruction of the neutrino energy and direction. If $\nu$ and $\bar{\nu}$ distributions are measured independently, we would have $\left|S_{\text {tot }}^{\nu}\right|=46.8,\left|S_{\text {tot }}^{\bar{\nu}}\right|=43.8$, and the total significance $\left|S_{\text {tot }}\right|=\sqrt{\left|S_{\text {tot }}^{\nu}\right|^{2}+\left|S_{\text {tot }}^{\bar{\nu}}\right|^{2}}=64.1$. The latter is about 3 times larger than the total significance in the case when $\nu$ and $\bar{\nu}$ signals are not separated $\left|S_{\text {tot }}^{\nu+\bar{\nu}}\right|=23.7$, in agreement with our qualitative result in sect. III.

In the realistic case of partial separation of the $\nu$ and $\bar{\nu}$ signals, which takes place when $y$-information is included, and after the kinematical smearing the significance decreases strongly: down to 8.43 after one year (so that after 3 years of exposure we would have $\left|S_{\mathrm{tot}}^{3 \mathrm{yr}}\right|=$ 14.6). This number further reduces down to $\left|S_{\mathrm{tot}}^{3 \mathrm{yr}}\right| \approx 6.1$ 

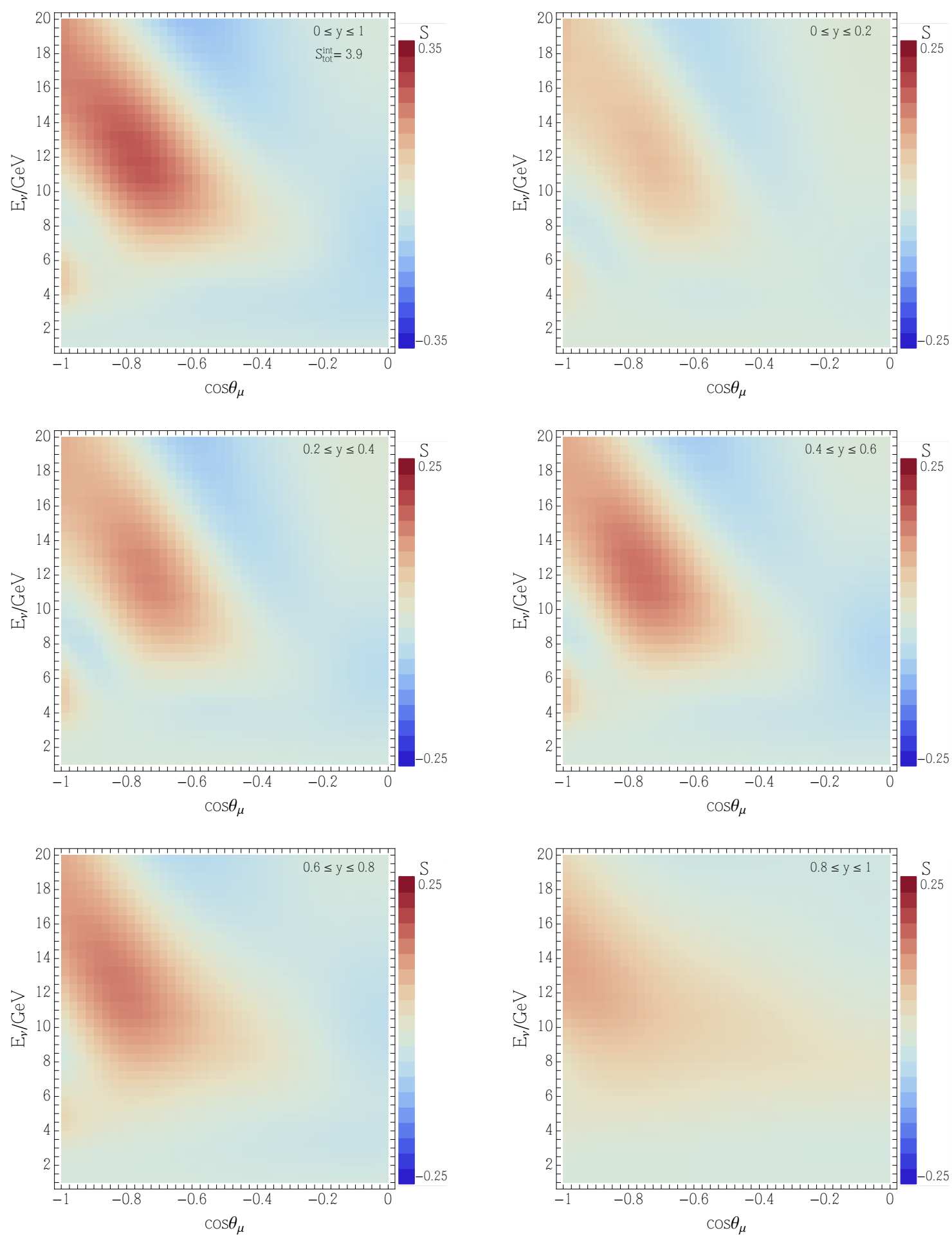

FIG. 4: The hierarchy asymmetry distribution in the $\left(E_{\nu}-\cos \theta_{\mu}\right)$ plane for different intervals of the inelasticity and for 1 year of exposure. The experimental smearing of the distributions was performed with the energy and angular resolutions $\sigma_{E}=\sqrt{0.7 E}, \psi_{0}=20^{\circ}$.

after the experimental smearing in our worst case scenario $\left(\sigma_{\mu, h}=\sqrt{0.7 E_{\mu, h}}, \sigma_{\psi}=40^{\circ}\right)$.

Systematic uncertainties likely play a relatively mild role in degrading these results, as the measurements are differential from neighboring locations (bins in $E_{\nu}$ and $c_{\mu}$ ), and the systematic uncertainties between neighbor- 

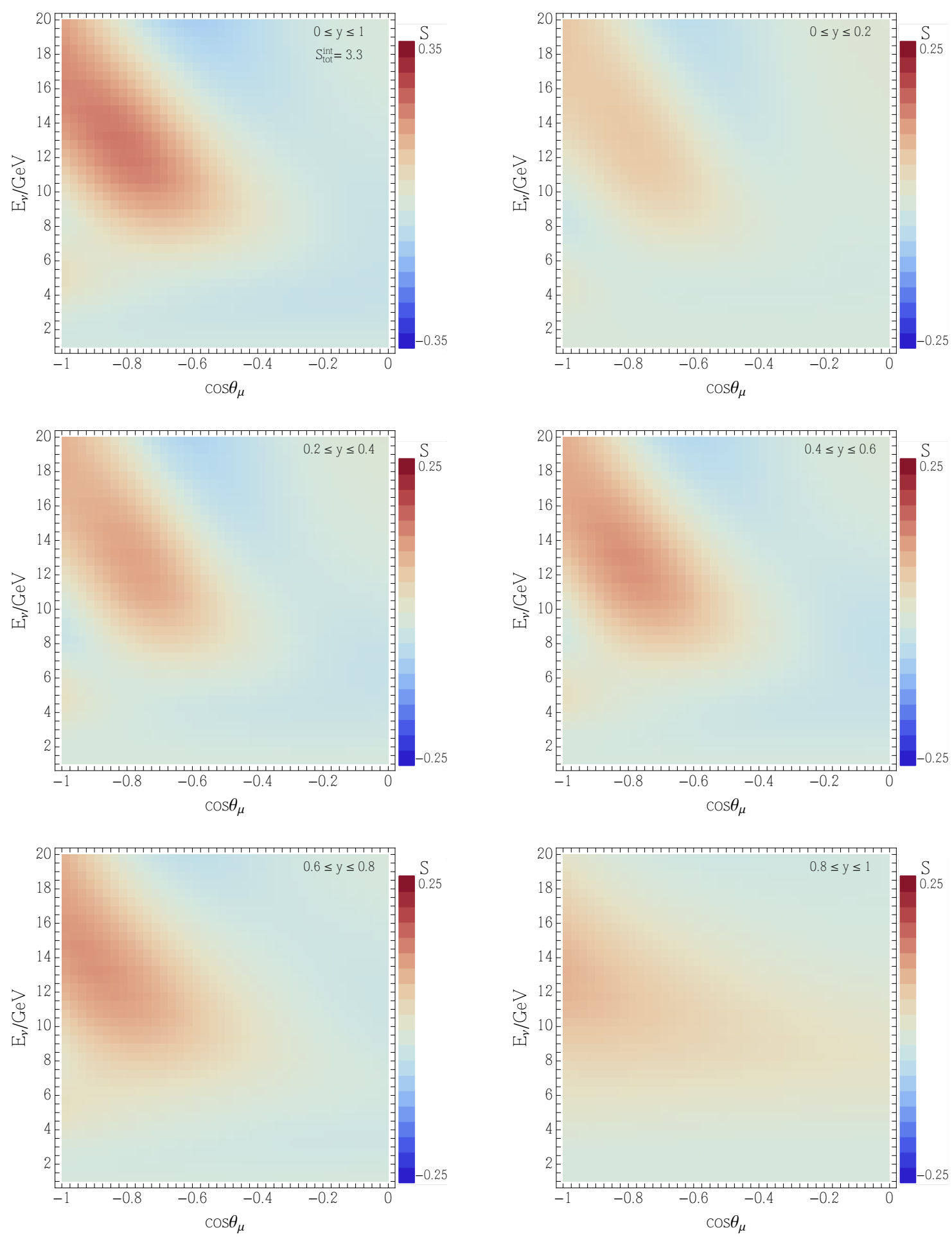

FIG. 5: The same as in Fig. 4 with $\sigma_{E}=\sqrt{0.7 E}$, and $\psi_{0}=40^{\circ}$.

ing bins with different asymmetries are strongly correlated. In addition, the $y$-distribution must be a superposition of the $y$-distributions of neutrino and of antineutrino events, strongly constraining its shape and providing information related to the systematic effect in the $y$-dimension. However, we have also found negligible degradation of the significance (about 1\%) if introduced as in 9, at level of $10 \%$. This is due to the small bin size of our oscillograms.

Fig. 7 shows the dependence of the significance on the 

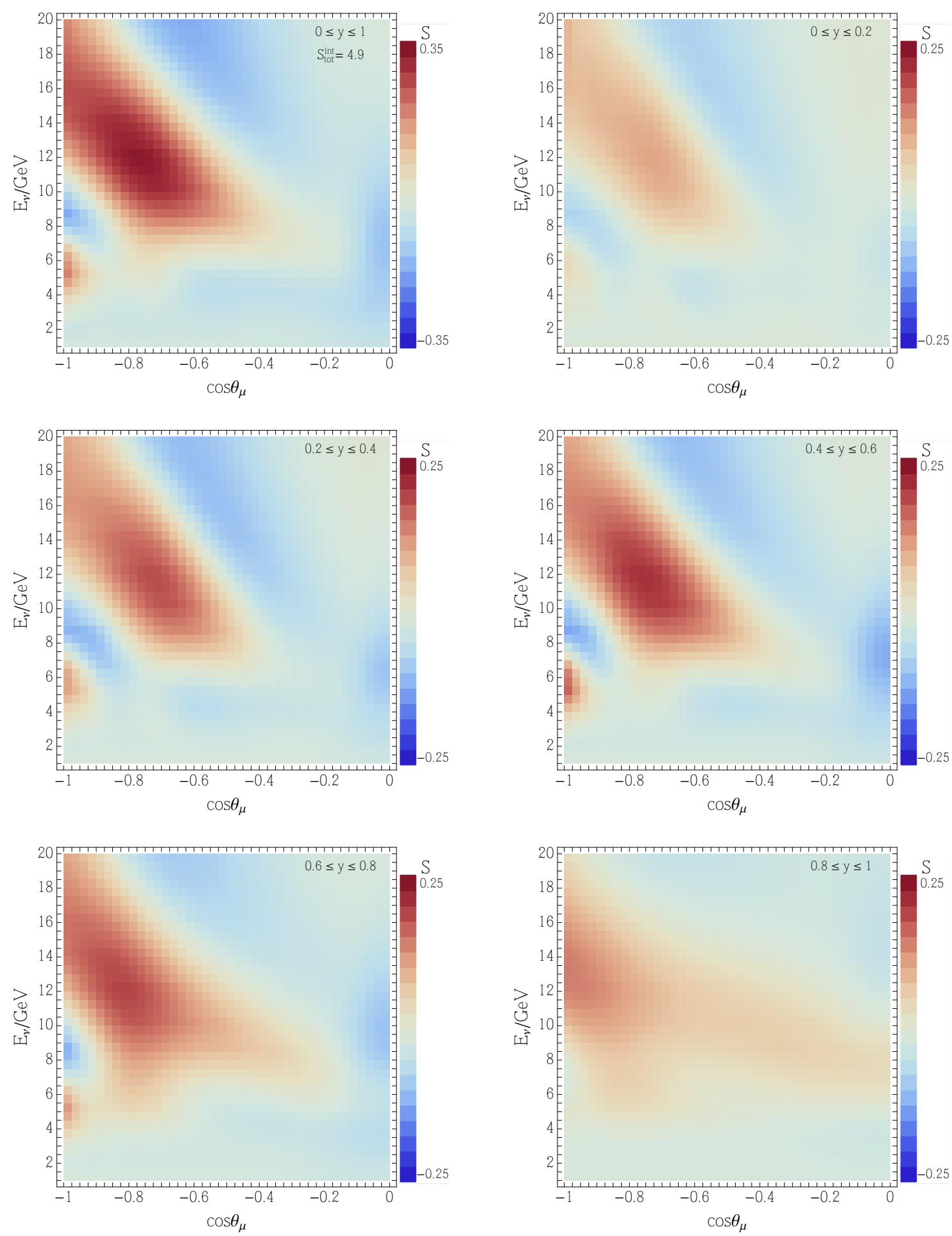

FIG. 6: The same as in Fig. 4 with $\sigma_{E}=\sqrt{0.35 E}$ and $\psi_{0}=10^{\circ}$.

upper limit of integration over $y$ for the case $\sigma_{\mu, h}=$ $\sqrt{0.35 E_{\mu, h}}$ and $\psi_{0}=20^{\circ}$ and for kinematical smearing only. The dashed curves are for $y$-integrated significances.

According to Fig. 7, the increase of the significance is sustained up to higher $y$ for curves including experimental smearing. This is due to the contribution to the asymmetry from larger $E_{\nu}$, whose relative importance in smearing decreases. Also the difference between $y$-differential and $y$-integrated significances is relatively 


\begin{tabular}{cc|ccc}
\hline$\sigma_{E}$ & $\psi_{0}$ & $\left|S_{\text {tot }}\right|$ & $\left|S_{\text {tot }}^{\text {int }}\right|$ & $\left|S_{\text {tot }}\right| /\left|S_{\text {tot }}^{\text {int }}\right|$ \\
\hline 0 & 0 & 8.43 & 7.11 & 1.19 \\
$\sqrt{0.35 E}$ & $10^{\circ}$ & 5.44 & 4.90 & 1.11 \\
$\sqrt{0.35 E}$ & $20^{\circ}$ & 5.10 & 4.66 & 1.10 \\
$0.3 \mathrm{E}$ & $20^{\circ}$ & 4.40 & 3.98 & 1.11 \\
$\sqrt{0.7 E}$ & $20^{\circ}$ & 4.19 & 3.87 & 1.08 \\
$\sqrt{0.7 E}$ & $40^{\circ}$ & 3.52 & 3.26 & 1.08 \\
\hline
\end{tabular}

TABLE I: The total significance of identification of the neutrino mass hierarchy for different experimental smearing scenarios and for 1 year of exposure. $S_{\text {tot }}$ refers to analysis with inelasticity, whereas $S_{\mathrm{tot}}^{\mathrm{int}}$ - for $y$-integrated distributions analysis. The upper line with $\sigma_{E}=\psi_{0}=0$ corresponds to the kinematical smearing only.

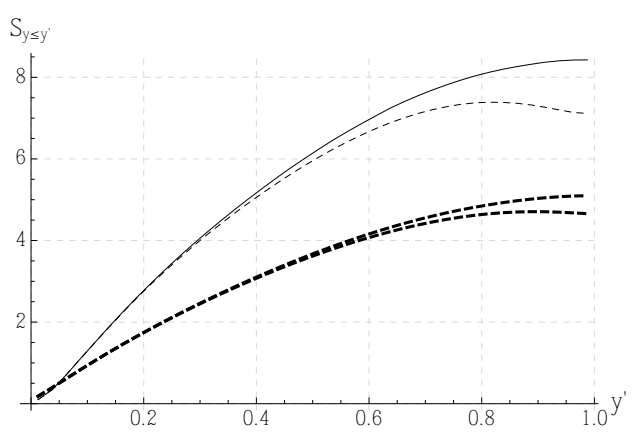

FIG. 7: Dependence of the significance on the upper limit of integration over $y$. Thin and thick curves respectively correspond to kinematical smearing only and the experimental smearing added. Solid and dashed curves respectively show $S_{\text {tot }}$ and $S_{\mathrm{tot}}^{\text {int }}$.

small after the experimental smearing.

\section{D. $\Delta m_{32}^{2}$ degeneracy}

The effect of an inversion of the mass hierarchy (especially at large energies) is rather similar to a shift of the oscillation probabilities in the energy scale (see 9]). This is equivalent to a change of $\Delta m_{32}^{2}$. Therefore the effect of hierarchy can be partly mimicked by a change of $\Delta m_{32}^{2}$. Indeed, the pattern of distribution of the quantity

$$
S^{\delta} \equiv \frac{N^{\mathrm{NH}}\left(\Delta m_{32}^{2}+\delta\right)-N^{\mathrm{NH}}\left(\Delta m_{32}^{2}\right)}{\sqrt{N^{\mathrm{NH}}\left(\Delta m_{32}^{2}\right)}}
$$

in $E_{\nu}-c_{\mu}$ plane is rather similar for certain values of the shift parameter, $\delta$, to the hierarchy asymmetry pattern:

$$
S^{\mathrm{MH}} \equiv \frac{N^{\mathrm{IH}}\left(\Delta m_{32}^{2}\right)-N^{\mathrm{NH}}\left(\Delta m_{32}^{2}\right)}{\sqrt{N^{\mathrm{NH}}\left(\Delta m_{32}^{2}\right)}} .
$$

Since $\Delta m_{32}^{2}$ is known with some error, this parameter degeneracy degrades the sensitivity to the mass hierarchy. To quantify the effect the following significance has been computed in [9]:

$$
S^{\mathrm{MH}-\delta} \equiv \frac{N^{\mathrm{IH}}\left(\Delta m_{32}^{2}+\delta\right)-N^{\mathrm{NH}}\left(\Delta m_{32}^{2}\right)}{\sqrt{N^{\mathrm{NH}}\left(\Delta m_{32}^{2}\right)}},
$$

where $\delta$ has been considered as a free parameter. This would correspond to $\mathrm{NH}$ as the true hierarchy and $\Delta m_{32}^{2}$ as the true value. The true distribution $N^{\mathrm{NH}}\left(\Delta m_{32}^{2}\right)$ is then fitted by IH distribution with arbitrary values of $\Delta m_{32}^{2}$. It has been found in [9] that the minimum $S_{\mathrm{min}}^{\mathrm{MH}-\delta}$ is reached for $\delta \approx-0.5 \sigma\left(\Delta m_{32}^{2}\right)$, where $\sigma\left(\Delta m_{32}^{2}\right)$ is the present $1 \sigma$ accuracy of determination of $\Delta m_{32}^{2}$ from the global fit [17. The minimal value (for 1 year of exposure and no inelasticity information) $S_{\min }^{\mathrm{MH}-\delta}=3.8$ should be compared with $S^{\mathrm{MH}-\delta}(\delta=0)=6.0$, thus showing reduction of the significance by a factor 1.6.

Future measurements at accelerators will reduce the error by factor of 2 , which means that no significant improvement is expected.

Let us show how information about inelasticity (or usage of 3D distributions) may help. As we mentioned before, the effect of a $\Delta m_{32}^{2}$ change is nearly the same for neutrinos and antineutrinos, whereas $y$-distributions are different.

We construct the distribution $S^{\delta \text {,int }}$ and the residual asymmetry plot $S^{\delta \text {,int }}-S^{\mathrm{MH} \text {,int }}$ which can be rewritten according to 40 and 41 as

$$
S^{\delta \text {,int }}-S^{\text {int }}=-\frac{N^{\mathrm{IH}}\left(\Delta m_{32}^{2}\right)-N^{\mathrm{NH}}\left(\Delta m_{32}^{2}-\delta\right)}{\sqrt{N^{\mathrm{NH}}\left(\Delta m_{32}^{2}\right)}} .
$$

After substitution $\left(\Delta m_{32}^{2}-\delta\right) \rightarrow \Delta m_{32}^{2}$ this residual asymmetry essentially coincides with the quantity $S^{\mathrm{MH}-\delta}$ 42 computed in 9 .

On the left panels of Figs. 8 and 9, we respectively show the plots for the asymmetry $S^{\text {int }}$ and for the residual asymmetry $S^{\delta \text {,int }}-S^{\text {int }}$ for an ideal detector after kinematical smearing only. We take $\delta \approx-0.5 \sigma\left(\left|\Delta m_{32}^{2}\right|\right)=$ $-6 \cdot 10^{-5} \mathrm{eV}^{2}$, which corresponds to the maximal degeneracy effect according to [9. On the right panels of Figs. 8 and 9 , we present respectively the asymmetry $S^{\text {int }}$ and the residual asymmetry $S^{\delta \text {,int }}-S^{\text {int }}$ after application of the experimental smearing. We used $\sigma_{E}=\sqrt{0.7 E}$, and $\psi_{0}=20^{\circ}$ as a realistic experimental resolution.

The total significance of determination of the mass hierarchy can be computed using the residual asymmetry as

$$
S_{\mathrm{tot}}^{\mathrm{int}}(\mathrm{MH}-\delta)=\sqrt{\int \mathrm{d} E_{\nu} \int \mathrm{d} c_{\mu}\left(S^{\delta, \text { int }}-S^{\text {int }}\right)^{2}}
$$

for the distributions without $y$-information, and

$$
S_{\text {tot }}(\mathrm{MH}-\delta)=\sqrt{\int \mathrm{d} E_{\nu} \int \mathrm{d} c_{\mu} \int \mathrm{d} y\left(S^{\delta}-S\right)^{2}}
$$

with $y$-distribution. For $\delta \approx-0.5 \sigma\left(\left|\Delta m_{32}^{2}\right|\right)$ and after 1 year exposure we obtain $S_{\mathrm{tot}}^{\text {int }}(\mathrm{MH}-\delta)=4.23$. This should be compared with $S_{\mathrm{tot}}^{\mathrm{int}}=7.11$ without degeneracy effect (see Table II). So, the degeneracy effect found in such a way corresponds closely to the one found in 9]. With the $y$-distribution, we obtain $S_{\mathrm{tot}}^{\mathrm{MH}-\delta}=6.03$ $\left(S_{\text {tot }}=8.43\right)$. Thus, the total significance is enhanced by $\sim 43 \%$ using the inelasticity. 

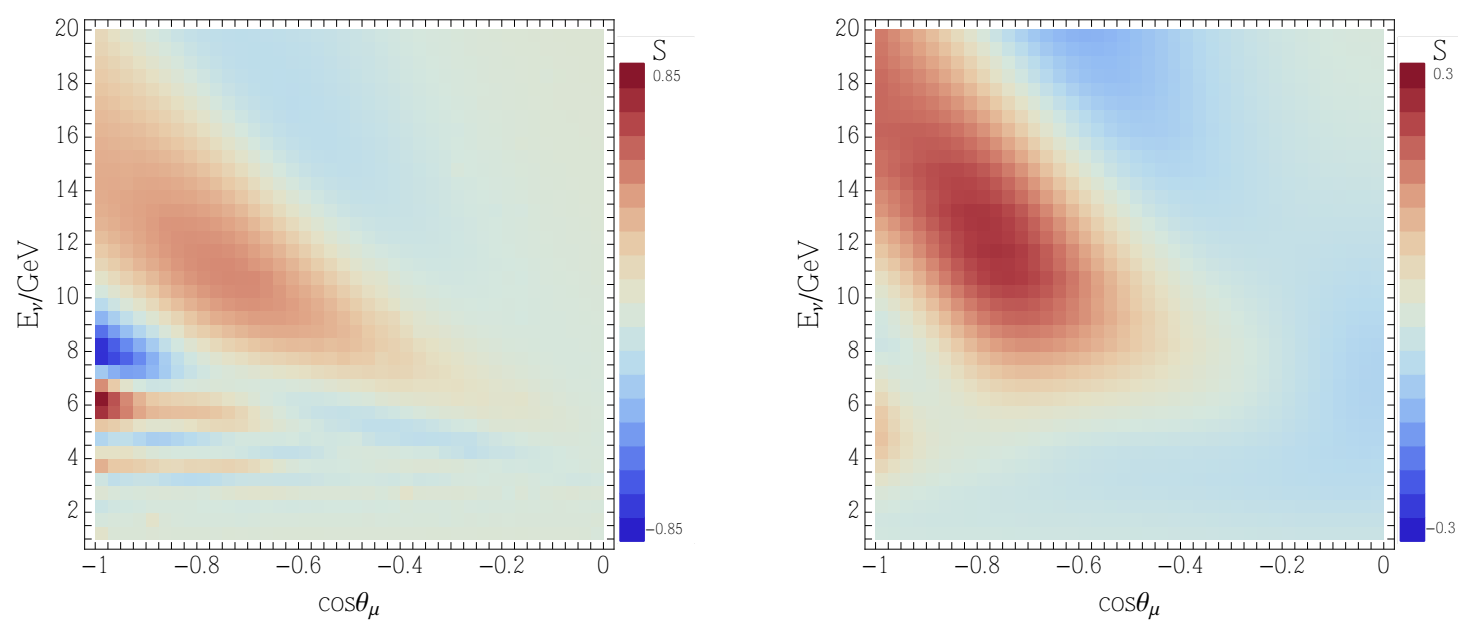

FIG. 8: Asymmetry plots $S^{\text {int }}$ with the kinematical smearing only (left) and after application of the experimental smearing $\sigma_{E}=\sqrt{0.7 E}, \sigma_{\psi}=20^{\circ} \sqrt{\frac{m_{\mathrm{p}}}{E_{\mu}}}$ (right), for 1 year of exposure.
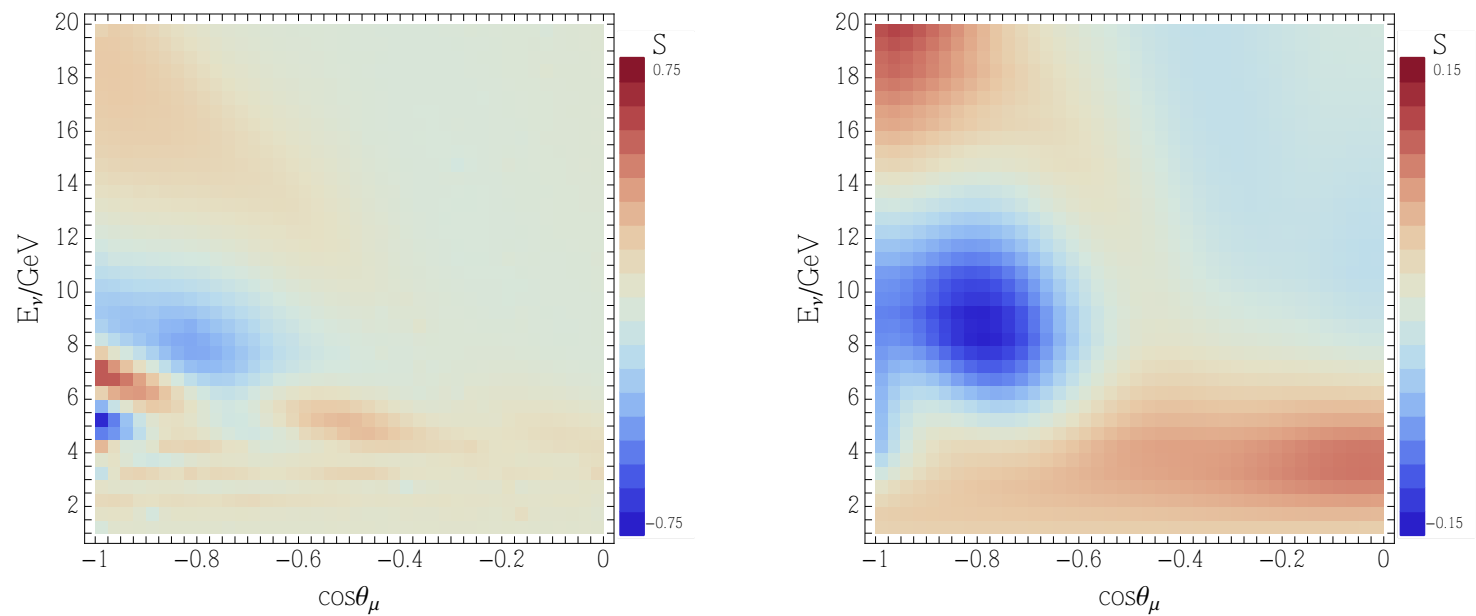

FIG. 9: Residual asymmetry plots $\left(S^{\delta, i n t}-S^{\text {int }}\right)$ with the kinematical smearing only (left) and after application of the experimental smearing $\sigma_{E}=\sqrt{0.7 E}, \sigma_{\psi}=20^{\circ} \sqrt{\frac{m_{\mathrm{p}}}{E_{\mu}}}$ (right), for 1 year of exposure. find

After the experimental smearing described above we

$$
S_{\text {tot }}^{\text {int }}(\mathrm{MH}-\delta)=1.93, \quad S_{\text {tot }}(\mathrm{MH}-\delta)=2.42
$$

(without degeneracy we would have $S_{\text {tot }}^{\text {int }}=3.87$ and $S_{\text {tot }}=4.19$, see Table I . The significance enhancement is reduced to about $25 \%$.

These results mean that the necessary exposure to ascertain the mass hierarchy with an ideal detector is a factor 2 larger if $y$ is not exploited. For the detector with the above mentioned experimental resolutions, this factor is not as large but still significant, about 1.55. The additional relative power of the inelasticity, as we already noticed from numbers in Table $\mathbb{\mathbb { T }}$, is greater, when detector resolutions are better.

\section{DISCUSSION AND CONCLUSIONS}

Multi-megaton scale under-ice and under-water detectors of atmospheric neutrinos with low (few GeV's) energy thresholds open up new possibilities for the determination of neutrino properties. This includes the neutrino mass hierarchy, the deviation of the 2-3 mixing from maximal and high accuracy measurement of $\Delta m_{32}^{2}$.

With a dense array of optical modules, it will be possible to identify different atmospheric neutrino events, and in particular, the $\nu_{\mu} \mathrm{CC}$ events and determine their characteristics. For the $\nu_{\mu}$ events, it will be possible to measure not only the energy and the direction of the muon, but also the energy of accompanying hadron cascade. The latter then determines the inelasticity. With 
$y$, one can construct the three-dimensional distributions of events in $\left(E_{\nu}, \cos \theta_{\mu}, y\right)$.

In this paper, we have explored various improvements of sensitivity to the mass hierarchy, which will be possible with the inclusion of the inelasticity in the analysis. The results can be summarized in the following way.

1. Inelasticity measurements provide a certain sensitivity to separate signals from neutrinos and antineutrinos. This, in turn, reduces the cancellation of the neutrino and antineutrino contributions to the hierarchy as well as to CP-violation effects.

We find that, in the ideal case of complete separation or independent measurement of $\nu$ and $\bar{\nu}$ signals, the significance of the hierarchy determination increases by factor $\sim 2.2-3$. However, finite accuracy of the separation (extraction of the parameter $\alpha$ ) reduces the effect down to $(20-30) \%$. The best separation is in the range of large $y$ where, however, the angular smearing becomes strong and effect of mass hierarchy is averaged out.

2. The selection of events with small $y$ allows one to reduce the angle between the neutrino and muon directions and therefore reduce the kinematical smearing, which is very strong at low energies in the resonance region where effect of mass hierarchy at the probability level is the biggest one. However, for small $y$, the effective separation of the $\nu$ and $\bar{\nu}$ contributions worsen, and moreover, the statistics decreases with cut in $y$. So, for fixed exposure, the overall gain is rather modest.

Separation of the $\nu$ and $\bar{\nu}$ improves with increase of $y$, while the neutrino angle reconstruction improves with decrease of $y$. Therefore the analysis of these improvements should be done simultaneously. This requires study of the 3D distributions of events, which takes into account both separation and reduction of kinematical smearing automatically.

3. We have computed the 3D oscillograms of the $\nu_{\mu}$ events with the kinematical smearing (for this the kinematics of the $\nu_{\mu}$ CC-interactions has been taken into account precisely). We then found the 2D asymmetry distribution in the $E_{\nu}-\cos \theta_{\mu}$ plane for different intervals of $y$. The main contribution to the identification of the hierarchy follows from the intermediate range $y=0.3-0.7$, and the contributions from intervals $y=0.8-1.0$ and $y=0-0.2$ are small. The inelasticity enhances the total significance of determination of the mass hierarchy by about $20 \%$, which is consistent with our semi-qualitative analysis provided that a slight decrease of $\gamma$ is achieved.

4. We then performed smearing of the distributions over the observables: the energies of muon and cascade as well as the angle of muon. We used the Gaussian smearing functions assuming different widths and their dependences on energy. The experimental smearing further diminishes the total significance by factor $1.5-2.4$ depending on the energy and angular resolutions. The inclusion of the inelasticity leads to an increase of the total significance by $(8-11) \%$ after application of the experimental smearing: The stronger the smearing, the weaker the significance increase.

5. Inversion of the mass hierarchy and variations of other parameters have different effects on the $y$-distribution of events. This means that inelasticity measurements will alleviate the degeneracy of the hierarchy with $\theta_{23}$ and $\Delta m_{32}^{2}$. Without $y$ distribution the degeneracy with $\Delta m_{32}^{2}$ reduces the significance by factor $\sim 1.7$. The inelasticity measurements increase the total significance by $43 \%$ before the experimental smearing and by $25 \%$ with a specific reasonable experimental smearing scheme.

6. The mass hierarchy and the systematic errors affect the $y$ distribution differently. Therefore measurements of inelasticity will likely help to reduce the impact of systematic uncertainties.

7. The contamination of the $\nu_{\mu}$ event sample with other flavors leads to a suppression of the oscillation effects. The selection of events with not too large $y$ will help discriminate $\nu_{\mu} \mathrm{CC}$ events from event of other types and therefore mitigate the loss of features in the oscillatory pattern.

8. All in all, we expect that the inclusion of the inelasticity of the interaction in the analysis will increase the significance by $(20-50) \%$, which is equivalent to an increase of the exposure time or effective volume by factor $1.5-2$.

9. It is not excluded that more sophisticated analysis will lead to even stronger enhancement effect.

The next step in enhancement of the discovery potential of the Multi-megaton scale detectors could be related to some information about the direction of the cascade using detailed time information about development of event. Also the inclusion of other type of events (cascades without muons) in the analysis will reinforce the discovery potential.

The inclusion of the inelasticity as an ingredient in the data analyses may become necessary in order to unambiguously conclude on the mass hierarchy in the near future.

\section{Acknowledgments}

M. Ribordy is supported by the Swiss National Research Foundation under the grant PP002-114800. We thank S. Razzaque for providing us the raw neutrino oscillograms used in [9] and for discussions in the initial phase of this project and R. Bruijn for his help with the GENIE simulation in order to understand the light output and the point-like nature of the low energy cascades. 


\section{References}

[1] E. K. Akhmedov, M. Maltoni and A. Y. Smirnov, JHEP 0705 (2007) 077 hep-ph/0612285; JHEP 0806 (2008) 072 arXiv:0804.1466 [hep-ph]]; Phys. Rev. Lett. 109 (2012) 091801 arXiv:1203.6012 [hep-ph]].

[2] M. Blennow and A. Yu. Smirnov, Advances in High Energy Physics, review article 972485.

[3] D. J. Koskinen, Mod. Phys. Lett. A 26 (2011) 2899.

[4] R. Abbasi et al. [IceCube Collaboration], arXiv:1109.6096 [astro-ph.IM].

[5] P. Coyle et al. [The Km3Net collaboration], contribution to the European Strategy Preparatory Group Symposium, September 2012 Krakow, Poland.

[6] O. Mena, I. Mocioiu and S. Razzaque, Phys. Rev. D 78, 093003 (2008) arXiv:0803.3044 [hep-ph]].

[7] E. Fernandez-Martinez, G. Giordano, O. Mena and I. Mocioiu, Phys. Rev. D 82, 093011 (2010) arXiv:1008.4783 [hep-ph]].

[8] J. Tang and W. Winter, JHEP 1202 (2012) 028 arXiv:1110.5908 [hep-ph]].

[9] E. K. Akhmedov, S. Razzaque and A. Y. Smirnov, JHEP 1302 (2013) 082, arXiv:1205.7071 [hep-ph].

[10] D. Franco, C. Jollet, A. Kouchner, V. Kulikovskiy, A. Meregaglia, S. Perasso, T. Pradier and A. Tonazzo et al., arXiv:1301.4332 [hep-ex].

[11] S. K. Agarwalla, T. Li, O. Mena and S. Palomares-Ruiz, arXiv:1212.2238 [hep-ph].

[12] M. Ribordy, in G. Bellini and L. Ludhova (Eds.). (2013). Neutrino Physics and Astrophysics, Ios PressInc. (ISAPP school proceedings, course CLXXXII, Varenna, Italy, 2011 arXiv:1205.4965 [astro-ph.HE]]).

[13] M. Ribordy, Nucl. Instrum. Meth. A 574 (2007) 137 [arXiv:0611604 [astro-ph]].

[14] H. L. Lai et al., Eur. Phys. J. C12 (2000) 375.

[15] C. Andreopoulos et al., Nucl. Instrum. Meth. A614 (2010) 87.

[16] M. Salathe, M. Ribordy and L. Demirors, Astropart. Phys. 35 (2012) 485 arXiv:1106.1937 [astro-ph.IM]].

[17] G. L. Fogli, E. Lisi, A. Marrone, D. Montanino, A. Palazzo and A. M. Rotunno, Phys. Rev. D 86 (2012) 013012 arXiv:1205.5254 [hep-ph]]; M. C. GonzalezGarcia, M. Maltoni, J. Salvado and T. Schwetz, JHEP 1212 (2012) 123 arXiv:1209.3023 [hep-ph]]; D. V. Forero, M. Tortola and J. W. F. Valle, Phys. Rev. D 86 (2012) 073012 arXiv:1205.4018 [hep-ph]].

\section{Appendix A: Variable change $\phi \rightarrow c_{\nu}$}

Consider a muon with vector $\boldsymbol{\mu}=\left(s_{\mu}, 0, c_{\mu}\right)$. The matrix associated to the rotation $\phi$ around the $\mu$ axis is

$$
R_{\mu}(\phi)=\left(\begin{array}{ccc}
c_{\phi} c_{\mu}^{2}+s_{\mu}^{2} & -c_{\mu} s_{\phi} & s_{\phi / 2}^{2} s_{2 \mu} \\
c_{\mu} s_{\phi} & c_{\phi} & -s_{\phi} s_{\mu} \\
s_{\phi / 2}^{2} s_{2 \mu} & s_{\phi} s_{\mu} & c_{\mu}^{2}+c_{\phi} s_{\mu}^{2}
\end{array}\right) .
$$

We consider a neutrino vector $\nu_{0}$ at an angle $\beta$ also in the plane $x-z$. A possible vector is $\boldsymbol{\nu}_{0}=\left(s_{\nu_{0}}, 0, c_{\nu_{0}}\right)$, with $\theta_{\nu}=\theta_{\mu}+\beta$. We use the matrix to generate revolution vector set $\{\boldsymbol{\nu}(\phi)\}_{\phi}$ around the muon trajectory,

$$
\boldsymbol{\nu}(\phi)=R_{\mu}(\phi) \boldsymbol{\nu}_{0}=\left(\begin{array}{c}
c_{\phi} c_{\mu} s_{\beta}+s_{\mu} c_{\beta} \\
s_{\phi} s_{\beta} \\
-c_{\phi} s_{\mu} s_{\beta}+c_{\mu} c_{\beta}
\end{array}\right) .
$$

The $z$-component of the vector $\boldsymbol{\nu}$ can be associated to $c_{\nu}$, $c_{\nu}=-c_{\phi} s_{\mu} s_{\beta}+c_{\mu} c_{\beta}$ therefore $\mathrm{d} \phi=\mathrm{d} c_{\nu} / s_{\beta} s_{\mu} s_{\phi}$, with $s_{\beta} s_{\mu} s_{\phi}=\sqrt{s_{\mu}^{2} s_{\beta}^{2}-\left(c_{\beta} c_{\mu}-c_{\nu}\right)^{2}}$.

\section{Appendix B: Smearing function for $y$}

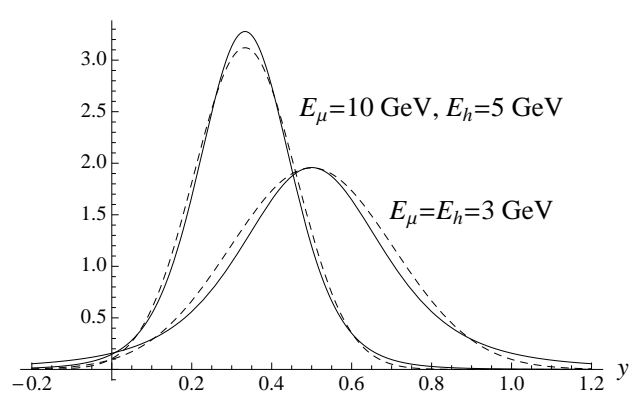

FIG. 10: The reconstructed $y$-distribution for two different sets of $\left\{E_{\mu}, E_{h}\right\}$ (numbers at the curves) and $\sigma_{\mu, h}=\sqrt{ } E_{\mu, h}$. The dashed curves correspond to the Gaussian approximation.

We use the notation $\Delta_{\mu} \equiv E_{\mu}-\tilde{E}_{\mu}, \Delta_{h} \equiv E_{h}-\tilde{E}_{h}$ and the simplified relation $y=E_{h} /\left(E_{\mu}+E_{h}\right)$. In order to obtain the $y$-distribution, we introduce $z \equiv E_{\mu} / E_{h}$, so that $y=1 /(1+z)$. Consequently, $z=1 / y-1$ and $\mathrm{d} z=-\mathrm{d} y / y^{2}$. Let us denote by $P_{z}(\tilde{z})$ the distribution of $\tilde{z}$. Then the $y$-distribution is given by

$$
P_{y}(\tilde{y})=\frac{1}{\tilde{y}^{2}} P_{z}(1 / \tilde{y}-1)
$$

In turn, the distribution of ratio $z$ can be found from

$$
P_{z}(\tilde{z})=\int g_{h}\left(\tilde{E}_{h}, E_{h}\right) g_{\mu}\left(\tilde{E}_{\mu}, E_{\mu}\right) \delta\left(\frac{E_{\mu}}{E_{h}}-\tilde{z}\right) \mathrm{d} E_{h} \mathrm{~d} E_{\mu}
$$


The integration gives

$$
\begin{aligned}
& P_{z}(\tilde{z})= \frac{e^{-\frac{\Delta_{h}^{2}}{2 \sigma_{h}^{2}}-\frac{\Delta_{\mu}^{2}}{2 \sigma_{\mu}^{2}}}}{2 \pi\left(\sigma_{h}^{2}+\sigma_{\mu}^{2} \tilde{z}^{2}\right)^{2}\left|\Delta_{\mu} \sigma_{h}^{2}+\Delta_{h} \sigma_{\mu}^{2} \tilde{z}\right|} \\
& \times\left(2 \sigma_{h} \sigma_{\mu}\left(\sigma_{h}^{2}+\sigma_{\mu}^{2} \tilde{z}^{2}\right)\left|\Delta_{\mu} \sigma_{h}^{2}+\Delta_{h} \sigma_{\mu}^{2} \tilde{z}\right|\right. \\
&+\quad \sqrt{2 \pi} e^{\frac{\left(\Delta_{\mu} \sigma_{h}^{2}+\Delta_{h} \sigma_{\mu}^{2} \tilde{z}\right)^{2}}{2 \sigma_{h}^{2} \sigma_{\mu}^{2}\left(\sigma_{h}^{2}+\sigma_{\mu}^{2} \tilde{z}^{2}\right)}}\left(\Delta_{\mu} \sigma_{h}^{2}+\Delta_{h} \sigma_{\mu}^{2} \tilde{z}\right)^{2} \\
&\left.\times \quad \sqrt{\sigma_{h}^{2}+\sigma_{\mu}^{2} \tilde{z}^{2}} \operatorname{erf}\left(\frac{\left|\Delta_{\mu} \sigma_{h}^{2}+\Delta_{h} \sigma_{\mu}^{2} \tilde{z}\right|}{\sqrt{2} \sigma_{h} \sigma_{\mu} \sqrt{\sigma_{h}^{2}+\sigma_{\mu}^{2} \tilde{z}^{2}}}\right)\right) .
\end{aligned}
$$

The $y$ distribution obtained in this way is nearly Gaussian in most cases of interests. It starts to deviate from Gaussian, showing enhanced tails, when $E_{\mu}$ and $E_{h}$ are both small. This is illustrated Fig. 10 for the energy resolution widths $\sigma_{\mu, h}=\sqrt{ } E_{\mu, h}$. 